\title{
On the Role of the Nonlocal Hartree-Fock (HF) Exchange in Narrow-Band Materials
}

\author{
Michael C. Böhm \\ Institut für Physikalische Chemie, Physikalische Chemie III, Technische Hochschule Darmstadt
}

Z. Naturforsch. 40 a, 195-221 (1985); received December 17, 1984

Dedicated to Professor Dr. A. Weiss on the Occasion of his 60th Birthday

\begin{abstract}
The influence of the nonlocal Hartree-Fock (HF) exchange in narrow-band materials with finite band gaps is analyzed. The mean-field Hamiltonian in the crystal orbital (CO) basis contains two $k$-dependent matrix elements responsible for the dispersion of the one-electron levels: the classical tight-binding integrals (kinetic energy of the electrons) that decay exponentially as well as the (non)local exchange. The asymptotic behavior of the two-electron potential is determined by the fall-off of the intercell bond-order matrices; their range exceeds significantly the spatial extension of the $k$-dependent one-electron integrals. The analytic structure of the HF dispersions of narrow-band systems (weak intercell interactions) is largely influenced by the magnitude of the Fermi-correlation beyond the direct neighbors. The associated $\varepsilon(k)$ curves differ strongly from idealized tight-binding relations. The bands are broadened and show enhanced energy gradients in certain domains of $k$-space. Nonlinearities in the $\varepsilon(k)$ relations are a direct consequence of finite neighbor's approximations adopted for the evaluation of the lattice sums. The analytic structures of such HF bands are intermediate between idealized tight-binding relations of covalent solids, on one side, and HF dispersions of metals in (nearly-)free electron-gas approximations, on the other, that show divergent $\varepsilon(k)$ gradients at the Fermi level. The exchange influence in insulating narrow-band materials is restricted to the filled one-particle space; this is demonstrated by a perturbational analysis. The crucial importance of reliable numerical integration procedures for the determination of the intercell bond-order matrices is pointed out. Standard techniques may lead to artificial periodicities pretending unphysical decay properties of the electronic exchange. Dispersion patterns of a simple one-orbital model are analyzed as a function of the mutual strength of the kinetic hopping integrals and the HF exchange as well as the spatial extension of the $k$-dependent two-electron potential. The validity of the theoretical expectations deduced from simple model calculations is studied for two complex polymers. Important one-electron properties of one-dimensional (1D) porphyrinato nickel(II) derivatives ( 2 and 3 ) are investigated by means of semiempirical SCF (self-consistent-field) HF INDO (intermediate neglect of differential overlap) $\mathrm{CO}$ calculations. The lattice spacings of $\mathbf{2}$ and $\mathbf{3}$ differ by $0.31 \AA$. This geometrical distinction allows for an inversion of the relative importance of the $k$-dependent one- and two-electron contributions to the mean-field operator. The exchange influence on the width of the HF dispersion in 3 exceeds the one-electron part by nearly one order of magnitude. It is shown that band structure properties of narrow-band systems are neither properly described by one-electron models of the Wolfsberg-Helmholtz-type nor by bare (unscreened) HF dispersions. The width of a mean-field band calculated within a nonlocal exchange approximation has to be corrected for quasi-particle (QP) interactions beyond the HF scheme (i.e., long-range and short-range correlations and relaxations) as well as for electron (optical) phonon interactions. The phononic coupling leads to a narrowing of the band width via Franck-Condon-like vibrational overlaps; this part is independent of the theoretical details of the electronic structure investigation. Important physical consequences of the exchange-control in narrow-band solids are shortly discussed.
\end{abstract}

\section{Introduction}

The crystal orbital (CO) formalism as derived on the basis of the tight-binding approximation has become a powerful computational tool for the investigation of the electronic structures of low-dimensional materials and classical solid-state systems

Reprint requests to Dr. M. C. Böhm, Institut für Physikalische Chemie, Physikalische Chemie III, Technische Hochschule Darmstadt, Petersenstr. 20, D-6100 Darmstadt, West Germany.
$[1,2]$. The predictive capabilities as well as the numerical stabilities of ab initio and semiempirical $\mathrm{CO}$ variants have been reviewed by several authors $[2-8]$. There are two main sources leading to computational problems in band structure investigations which are both associated to the proper treatment of long-range (LR) summations in self-consistentfield (SCF) Hartree-Fock (HF) CO studies. The first one must be traced back to the various classical electrostatic energies entering the mean-field Hamiltonian in the crystal orbital basis (i.e., elec-

0340-4811/ $85 / 0300-0195 \$ 01.30 / 0$. - Please order a reprint rather than making your own copy. 
tron-electron and core-core repulsions, electron-core attraction). These elements can be combined to rapidly convergent lattice sums in covalent materials fulfilling the condition of charge neutrality. In ionic crystals, on the other side, the evaluation of the Coulomb potential within the HF scheme is similar to summations of the Madelung-type [9]. The second source responsible for the aforementioned problems is related to the convergence properties of the exchange energy giving rise to a $\cos (k j)$-dependence of the matrix elements of the HF operator. $k$ is the convenient wave vector characterizing the translational symmetry of the polymer or solid and $j$ stands for a cell index $(j=0$, reference cell in the origin, $j= \pm 1, \pm 2$, etc., $N_{i}$, first, second, etc., $N_{i}^{\prime}$ th nearest neighboring unit).

The asymptotic behaviour of the exchange potential and certain energetic consequences of realspace truncation criteria have been analyzed in simple model solids. The theoretical contributions can be divided into analytical studies limited to extremely plain systems (e.g. one- or two-orbital models defined within the Hückel approximation), on one side, and numerical investigations, on the other, which allow for the treatment of real solids in the framework of ab initio or semiempirical crystal Hamiltonians. The purely analytical contributions were often restricted to one-dimensional (1D) chains with half-filled bands. Cižek et al. were the first who analyzed modifications in the matrix elements of a PPP (Pariser-Parr-Pople) Hamiltonian via nonclassical exchange contributions [10]. Ukrainski investigated the exchange asymptotics of a oneorbital one-electron model and found a $(-1)^{j} j^{-2}$ decay of the exchange summation [11]. Furthermore he pointed out that divergent energy gradients, (1),

$$
\nabla_{\varepsilon}(k)=(\partial \varepsilon(k) \partial k)_{k= \pm \pi} \rightarrow \infty
$$

occur at the Brillouin zone leading to vanishing density of states (DOS) distributions $N(E)$. This logarithmic singularity at $k= \pm \pi$ has been known for a long time for metals in free and nearly-free electron-gas approximations in the HF scheme [12]. A reinvestigation of [11] and an extension to any solids with half-filled bands has been published by Monkhorst [13]. Simplified relations quantifying the exchange asymptotics in plain 1D chains with completely filled and half-filled bands have been derived by Monkhorst and Kertész [14]. These authors were able to show that the asymptotic decay of the ex- change energy is determined by the (intercell) density matrices $D_{\mu v}^{ \pm}(j)$ and can be expressed by means of (2) (insulating materials) and (3) (halffilled bands), respectively:

Filled bands:

$$
\lim _{j \rightarrow \infty} D_{\mu v}^{ \pm}(j)=j^{-1 / 2}(-\varrho)^{j},
$$

Half-filled bands:

$$
\lim _{j \rightarrow \infty} D_{\mu v}^{ \pm}(j)=(1 / 2 \pi)(-1)^{j} /|j| .
$$

Equations (2) and (3) are valid for a simple twoband model defined within a Hückel-type approximation. $\varrho$ in (2) is a complicated function and contains the band gap-band width ratio as well as the magnitude of hopping matrix elements between adjacent lattice sites as parameters. The divergent influence of the HF exchange in metallic and insulating systems had been recognized also by Piela et al. [5]. Reference [5] in addition gives a comparison of the exchange potential in finite and infinite Fermion systems.

Numerical studies on the influence of the exchange on the total energy of $1 \mathrm{D}$ chains have been reported over the past decade. One of the first contributions had been given by Fujita and Imamura [15]. Subsequent calculations based on ab initio $\mathrm{CO}$ procedures showed alternatively pronounced long-range effects of the exchange potential $[2,3$, $5-8,16-18]$ or the absence of significant LR contributions (i.e. interactions beyond the third or fourth nearest neighbors) $[4,19]$. A sophisticated explanation of this contradiction is still missing.

It is the common omission of the aforementioned theoretical and computational contributions to the exchange energy problem within the SCF HF CO approximation that narrow-band materials of experimental interest have not been analyzed in any detail. An important property of these solids are small hopping matrix elements (i.e. kinetic energy of the electrons) between neighboring unit cells. This reduction of the tight-binding interaction should allow for significant modifications of the band energies $\varepsilon(k)$ (positions and analytic structures of the dispersion curves), $N(E)$ distributions and related properties (i.e., effective masses, group velocities, etc.) due to nonlocal exchange effects. The condition of finding small kinetic energy integrals (with their characteristic exponential decay) but simultaneously remarkable contributions via 
slowly convergent exchange summations should be fulfilled in low-dimensional materials with weak (covalent) interactions between the stacking units.

Suitable model systems for the investigation of the influence of the exchange energy in narrowband materials are one-dimensional organic metals (e.g., TTF/TCNQ systems), on one side, as well as recently synthesized organometallic polymers, on the other. The transition metal atoms in most of the latter materials are embedded into an "organic matrix" formed by the surrounding ligand moieties [20, 21]. Recent semiempirical SCF HF CO calculations of various $1 \mathrm{D}$ chains with transition metal sites indeed have shown remarkable deviations of the observed HF dispersions from $\varepsilon(k)$ relations expected for idealized tight-binding bands [22-29]. The crystal orbital investigations cited in [22-29] span a wide range of topologically different $1 \mathrm{D}$ organometallic materials and are based on an improved INDO (intermediate neglect of differential overlap) $\mathrm{CO}$ formalism [30] which has been designed to reproduce the results of time-consuming ab initio calculations [31]. Furthermore it should be mentioned that band structure properties (e.g., band widths, $\varepsilon(k)$ relations) of transition metal species with macrocyclic $\pi$ ligands as derived by the above mentioned semiempirical SCF HF CO scheme (including exchange terms) differed significantly from the computational results of one-electron calculations of the Wolfsberg-Helmholtz (WH) or extended Hückel (EH) type in the nearest neighbor's approximation [32, 33]. The latter tight-binding variants are of course restricted to approximations of the kinetic energy of the full crystal Hamiltonian.

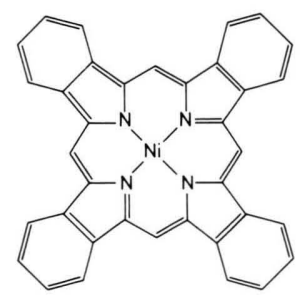

1

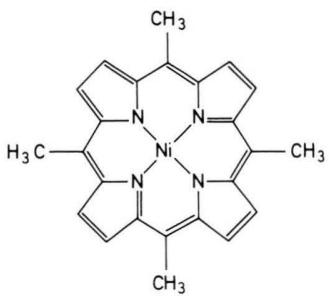

2

It is the purpose of the present article to investigate the influence of the exchange potential on the band structure properties of narrow-band materials. For the numerical calculations we have adopted the improved SCF HF INDO CO scheme described in [30]. The employed organometallic model systems are members of the porphyrinato nickel(II) series (see Fig. 1) [34-37]. These 1D chains have focused particular attention in the class of molecular metals containing organometallic stacking units as it is possible to influence the electronic conductivities of partly oxidized derivatives, their charge carrier properties and thus also the "correlation strength" $U / 4 t$ over wide ranges via modifications in the organic $\pi$ skeleton. The ratio $U / 4 t$ originally had been defined in the framework of the phenomenological Hubbard Hamiltonian [38-40] where $U$ stands for the on-site (Coulomb) repulsion and $4 t$ for the width of a simple tight-binding band ( $t$ : hopping integral). The correlation strength is enlarged for $U / 4 t \rightarrow \infty$ while delocalization effects (kinetic energy of the electrons) dominate in the limit $U / 4 t \rightarrow 0$.

Three important representatives of $\mathrm{Ni}(\mathrm{II})$ porphyrines are displayed in Figure 1. All materials are insulators in the absence of electron acceptors (e.g. halides) but highly conducting solids in the case of a partial charge transfer from the organometallic ribbon. Tetrabenzporphyrinato nickel(II), Ni(TBP) 1, crystallizes in form of strictly planar $\mathrm{Ni}(\mathrm{TBP})$ stacks with relatively short metal-metal separations of $3.220 \AA$ [34]. The magnetic susceptibility $\chi^{P}$ is Pauli-like and nearly temperature independent above $100 \mathrm{~K}$. The physical properties of the partly oxidized chain (e.g., temperature dependence of the

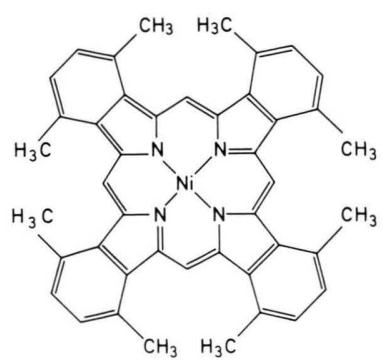

3

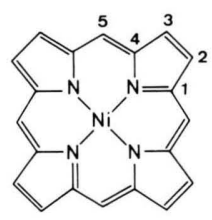

4

Fig. 1. 1: Tetrabenzporphyrinato nickel(II), Ni(TBP); 2: 5,10,15,20-tetramethylporphyrinato nickel(II), Ni(TMP); 3: 1,4,5,8,9,12,13,16-octamethyltetrabenzporphyrinato nickel(II), Ni(OMTBP); 4: porphyrinato nickel(II), Ni(P). The employed atomic numbering scheme is shown in the $\mathrm{Ni}(\mathrm{P})$ skeleton. 
conductivity) are still in line with the band picture. The four methyl groups of the small-ring system 5,10,15,20-tetramethylporphyrinato nickel(II), $\mathrm{Ni}$ (TMP) 2, cause a tetrahedral distortion around the central $3 \mathrm{~d}$ site and lead to an elongation of the $\mathrm{Ni}-\mathrm{Ni}$ distance to $3.466 \AA$ [35]. The nuclear distortion around $\mathrm{Ni}$ can be measured by the tilting angle between adjacent pyrrole rings which amounts to $17.5^{\circ}$ in 2 . The crystallographic point symmetry is thus reduced to $S_{4}$. The magnetic susceptibility of the conducting modification is once again metallike and temperature independent above the transition temperature of $28 \mathrm{~K}$. The increasing influence of the two-electron part (in comparison to $\mathbf{1}$ ), however, is monitored by a rather high $\chi^{P}$ value of 0.33 spins per stacking unit (1: 0.1 spins/ $\mathrm{Ni}(\mathrm{TBP})$ ). The third member in the porphyrinato series of Fig. 1 is the highly puckered $1,4,5,8,9,12,13,16$-octamethyltetrabenzporphyrinato nickel(II) polymer, $\mathrm{Ni}$ (OMTBP) 3, where the interdeck spacing amounts to $3.778 \AA[36,37]$. The pyrrole rings are now tilted by $40^{\circ}$. The measured conductivities $\sigma$ of $\mathbf{3}$ are $2-3$ orders of magnitude smaller than the $\sigma$ values of $\mathbf{1}$ and 2. Partially oxidized $\mathbf{3}$ shows a Curie-like susceptibility which indicates small interactions between the spins. The extrapolated antiferromagnetic exchange parameters $J$

$$
J=t^{2} / U
$$

are $J<3 \mathrm{~cm}^{-1}$ and $J<10 \mathrm{~cm}^{-1}$ for oxidized stacks with 0.36 and 0.97 electrons removed from Ni(OMTBP). The coincidence of Curie-like $\chi^{P}$ curves and significant conductivities had been explained in terms of correlated hopping motions of the charge carriers (i.e. polaronic transport processes) [36, 37]. 3 is thus the first realization of the "atomic limit $U / 4 t \rightarrow \infty$ of the Hubbard model" in the class of low-dimensional organic metals; an important physical consequence of this atomic limit is the decoupling of orbital and spin degrees of freedom [41].

This short presentation of some solid-state properties encountered in the partly oxidized porphyrinato nickel(II) series $\mathbf{1} \rightarrow \mathbf{2} \rightarrow \mathbf{3}$ explains immediately the challenging theoretical problem of investigating the importance and the spatial extension of the exchange energy in narrow-band systems by means of tight-binding variants defined within the mean-field approximation. In this context it is of general interest to gain deeper insight into the inter- relation between the distance-dependence of exchange summations, on one side, and band structure properties (e.g., band widths $\Delta \varepsilon$, analytical behaviour of $\varepsilon(k)$ curves, etc.), on the other. Equation (4), for instance, offers an access to "experimental" band widths $\Delta \varepsilon^{\exp }=4 t^{\exp }$ via measured $J$ parameters (from susceptibility data) and reliable estimates of the on-site repulsion $U$ which can be compared with theoretical findings $\left(\Delta \varepsilon^{\text {theor. }}\right)$. Although (4) contains no explicit assumption on the analytic structure of the partly filled band susceptible to the antiferromagnetic coupling it implies nevertheless that the width of the dispersion is exclusively determined by the one-electron part of the $\mathrm{CO}$ operator. Most of the convenient experimental procedures employed for the estimation of $\Delta \varepsilon$, however, are based on an even more stringent a priori assumption, i.e. the description of the relevant band states in terms of a simple tight-binding relation with a nearest neighbor's $\cos (k j)$-modulated hopping integral $(j=1)$. This condition is adopted in techniques where $t^{\exp }$ is related to spin susceptibilities $\chi^{P}[42-44]$, plasma frequencies $\omega_{P}$ [45] or measurements of the thermoelectric power $S$ [46]. Various extrapolations of $t^{\exp }$ parameters in one-dimensional metallomacrocycles, however, have shown that the experimental uncertainties are quite large, i.e. the derived $\Delta \varepsilon$ figures depend strongly on the adopted physical measuring quantity [47].

The band structure properties of suitable model polymers of $\mathbf{1}, \mathbf{2}$ and $\mathbf{3}$, respectively, have been studied previously $[25,26,48,49]$ by the INDO CO formalism of [30]. In these contributions we have adopted a fixed approximation for the expansion of the lattice sums (fifth nearest neighbor's scheme). The investigation of the solid-state electronic structure of $\mathbf{1}$ has shown that the width of the partly oxidized band is significantly larger than $1 \mathrm{eV} . \mathbf{1}$ is therefore no suitable candidate for the planned study. Preliminary tight-binding calculations of models of $\mathbf{2}$ and $\mathbf{3}$, on the other side, verified strong reductions in the width(s) of the conduction band(s) (i.e. valence band(s) of the unoxidized stacks) due to the elongation of the interdeck separation [48, 49]. Consequently we have restricted the present investigation of the exchange-influence in narrowband materials (formed by organometallic building blocks) to the latter two porphyrinato $\mathrm{Ni}(\mathrm{II})$ stacks $\mathbf{2}$ and 3. The unit cell dimensions of both $\mathrm{Ni}$ (II) polymers, however, are too large to allow for nu- 
merical crystal orbital studies within the SCF HF approximation. Therefore we adopted the simpler porphyrinato nickel(II) system, $\mathrm{Ni}(\mathrm{P}) \mathbf{4}$, as common $1 \mathrm{D}$ model, but used the relevant geometrical parameters of $\mathbf{2}$ and $\mathbf{3}$ (i.e., lattice spacing $c$, tetrahedral deformation around the central $\mathrm{Ni}$ sites, ligand geometries) in the actual $\mathrm{CO}$ calculations. The solid-state electronic structures of the porphyrinato nickel(II) systems are not discussed in larger detail in this context. For $\mathbf{1}$ we refer to [25, 26], for $\mathbf{2}$ and $\mathbf{3}$ to $[48-50]$.

The organization of the present manuscript is as follows: The theoretical background of the problem (exchange matrix elements in the SCF HF CO Hamiltonian and the total energy expression) is presented in section 2. Additionally possible error sources due to the numerical integration of the intercell bond-order matrices $D_{\mu \nu}^{ \pm}(j)$ are shortly touched. The subsequent part contains an analysis of simple, clear model calculations. The $\varepsilon(k)$ structure of a one-orbital model is investigated as a function of the decay characteristics of the exchange potential. Possible superposition-patterns of kinetic energy effects via a single (nearest neighboring) matrix element and LR contributions due to the exchange are also discussed. The plain examples embrace "sum curves" $\varepsilon(k)$ with opposite slopes of the kinetic energy and the nonlocal exchange and models showing possible interference effects between the two $k$-dependent contributions to the $\mathrm{CO}$ Hamiltonian. The computational conditions employed for the $\mathrm{CO}$ calculations of $\mathbf{2}$ and $\mathbf{3}$ are shortly described in Sect. 4 while the $j$-dependence of important band structure properties as well as of the total energies of the two low-dimensional nickel(II) chains are presented in the next paragraph. It will be shown that the electronic exchange leads to a broadening of the HF dispersions within the filled Fermi-sea as well as to nonlinearities in the $\varepsilon(k)$ curves. Band-narrowing mechanisms due to electronic correlations and electron-phonon coupling antagonizing the exchange potential are the subject of the following section. A summary and general conclusions are given in paragraph 7 .

\section{Theoretical Background}

The basic principles of the crystal orbital formalism have been described in the literature [51, 52] and are thus not reviewed in larger detail in the

present context. Only those aspects are recapitulated that are important in connection with the $k$-dependence of the exchange energy as well as the hopping matrix elements. The subsequent formulas refer to the INDO approximation as defined within the ZDO (zero differential overlap) hierarchy [30]. However, it can be shown that the exchange contributions encountered in the simplified INDO scheme are the leading terms of the electronic exchange in non-orthogonal (i.e. full overlap) $\mathrm{CO}$ expansions [14].

A LCAO (linear combination of atomic orbitals) representation of the canonical HF Bloch orbitals is given by

$$
\begin{aligned}
\psi_{l}(k)= & N^{-1 / 2} \sum_{j=0}^{(N-1)} \sum_{\mu=1}^{n} \\
& \cdot \exp (i j k) c_{\mu l}(k) \chi_{\mu}^{A_{j}}\left(r_{A}-j\right),
\end{aligned}
$$

where $l$ stands for a band index, $N$ for the number of unit cells and $\chi_{\mu}^{A_{j}}\left(r_{A}-j\right)$ for the $\mu$ 'th atomic orbital at the $A^{\prime}$ th site located in the $j^{\prime}$ th cell. The variational coefficients $c_{\mu l}(k)$ depend parametrically on the value of the crystal momentum $k$ and are derived by diagonalization of the Hermitean pseudoeigenvalue problem

$$
F(k) c(k)=\varepsilon(k) c(k) .
$$

Equation (6) is already the ZDO formulation of the general SCF HF CO eigenvalue problem. $F(k)$ symbolizes the Fock operator and the $\varepsilon(k)$ are the diagonal Lagrange multipliers forming the canonical HF bands. The Bloch vector $k$ is a continuous variable defined in the interval $-\pi \leqq k \leqq+\pi$ where the lattice constant $c$ has been identified with the unit vector of the solid.

The $k$-dependent eigenvalue problems are coupled via density matrices that are defined by

$$
\begin{aligned}
D_{\mu v}(k) & =2 \sum_{l=1}^{o c c} c_{\mu l}^{*}(k) c_{v l}(k) \\
D_{\mu v} & =N^{-1} \int_{0}^{2 \pi} D_{\mu v}(k) \mathrm{d} k \\
& =N^{-1} \int_{0}^{2 \pi}\left[2 \sum_{l=1}^{o c c} c_{\mu l}^{*}(k) c_{v l}(k)\right] \mathrm{d} k \\
& =N^{-1} \int_{0}^{2 \pi}\left[\sum_{l=1}^{o c c}\left(c_{\mu l}^{R}(k) c_{v l}^{R}(k)+c_{\mu l}^{I}(k) c_{v l}^{I}(k)\right)\right] \mathrm{d} k
\end{aligned}
$$




$$
\begin{aligned}
& D_{\mu v}^{ \pm}(j)= N^{-1} \int_{0}^{2 \pi} \exp ( \pm i j k) D_{\mu v}(k) \mathrm{d} k \\
&=2 N^{-1} \int_{0}^{\pi} \operatorname{Re}[\exp ( \pm i j k) \\
&\left.\quad \cdot\left(\sum_{l=1}^{\text {occ }} c_{\mu l}^{*}(k) c_{v l}(k)\right)\right] \mathrm{d} k .
\end{aligned}
$$

The formulas are valid for non-paramagnetic systems with completely filled bands leading to an integration interval from $-\pi$ to $+\pi$ (i.e. 0 to $2 \pi$ ) within the first Brillouin zone. The matrix $D_{\mu v}(k)$ is defined by the crystal orbital coefficients $c_{\mu l}^{*}(k)$ and $c_{v l}(k)$ of the filled bands. $D_{\mu v}$ in (8) is the bondorder matrix between the atomic orbitals $\chi_{\mu}\left(r_{A}\right)$ and $\chi_{v}\left(r_{B}\right)$ which are both localized in the reference cell. $D_{\mu v}^{ \pm}(j)$ is the Fourier-transform of $D_{\mu v}(k)$ associated to AO pairs $\chi_{\mu}\left(r_{A}\right) / \chi_{v}\left(r_{B}-j\right)$ that are $j$ unit cells apart.

The density matrices $D_{\mu v}$ and $D_{\mu v}^{ \pm}(j)$, respectively, are evaluated by numerical integration. The standard techniques are discrete summations or Simpson's quadrature. Other methods have been reported and compared by Delhalle [53, 54]; they are either based on the Gauss-Legendre quadrature or a Filon scheme. It is the drawback of the former (standard) integrations that they allow only for an approximation of the whole integrand in (9) but not of the $k$-dependent functions $D_{\mu v}(k)$ which are only known at the preselected number of $k$-points. The elements $D_{\mu v}(k)$ of $(7)$ are fully periodic:

$$
\begin{aligned}
& D_{\mu v}(k+2 n \pi)=D_{\mu v}(k), \\
& n= \pm 1, \pm 2, \pm 3, \ldots
\end{aligned}
$$

Under certain conditions it is however indispensible that convenient integration techniques (e.g., discrete summations, Simpson's formula) give rise to artificial periodicities in the intercell elements $D_{\mu v}^{ \pm}(j)$. Equation (9) can be rearranged into a sum of simple "harmonic motions" (integration procedure: Simpson's rule):

$D_{\mu v}^{ \pm}(j)=N^{-1}\left[\sum_{n=0}^{2 \pi} A_{\mu v}\left(k_{n}\right) \cos \left(k_{n} j \pm \Theta\right)\right]$.

The amplitudes $A_{\mu v}\left(k_{n}\right)$ are determined by proper combinations of the real and imaginary parts of the $\mathrm{CO}$ coefficients (see (12)). The phase factor $\Theta$ depends on the ratio of the two summations entering the equation

$$
\begin{aligned}
A_{\mu v}\left(k_{n}\right)= & \sum_{l=1}^{\mathrm{occ}}\left[\left(c_{\mu l}^{\mathrm{R}}\left(k_{n}\right) c_{v l}^{\mathrm{R}}\left(k_{n}\right)+c_{\mu l}^{\mathrm{I}}\left(k_{n}\right) c_{v l}^{\mathrm{I}}\left(k_{n}\right)\right) \mid\right. \\
& \left.\quad+\mid\left(c_{\mu l}^{\mathrm{R}}\left(k_{n}\right) c_{v l}^{\mathrm{I}}\left(k_{n}\right)-c_{\mu l}^{\mathrm{I}}\left(k_{n}\right) c_{v l}^{\mathrm{R}}\left(k_{n}\right)\right)\right] \\
= & \mid C^{\mathrm{R} \mathrm{RII}}+C^{\mathrm{R} I I \mathrm{R}} ; \\
\Theta= & C^{\mathrm{R} \mathrm{RII}} / C^{\mathrm{RIIR}} .
\end{aligned}
$$

It is obvious that $D_{\mu v}^{ \pm}(j)$, when derived via Simpson's rule, is a periodic function with respect to $j$ where the periodicity amounts to $2 k_{n} ; k_{n}$ is the number of $k$-points employed in the numerical integration. Equation (11) shows thus immediately that the relative errors of the integration are enhanced with increasing $j$-arrays relative to a given grid of $k$-figures (see below). This behaviour of the $D_{\mu v}^{ \pm}(j)$ elements is known phenomenologically for several years and lead to the formulation of qualitative rules, how to fix the number of neighboring unit cells to an employed $k$-grid $[4,15,53,54]$.

In order to come to a clear representation of the matrix elements of the Fock operator $F_{\mu v}(k)$ we divide the various integrals in the $\mathrm{AO}$ basis into $k$-dependent $\left(F K_{\mu v}(k)\right)$ and $k$-independent contributions $\left(F I_{\mu \nu}\right)$ :

$$
F_{\mu v}(k)=F K_{\mu v}(k)+F I_{\mu v} .
$$

Within the framework of the adopted INDO approximation we observe the relations (15) to (20) for the diagonal and off-diagonal elements of the mean-field Hamiltonian ( $\mathrm{R}$ symbolizes the real and I the imaginary part of $\left.F K_{\mu v}(k)\right)$ :

$$
\begin{aligned}
F I_{\mu \mu}= & H_{\mu \mu}+\sum_{B_{0}} V_{\mu_{0} B_{0}}^{A_{0} B_{0}}+\sum_{\lambda_{0}}^{A_{0}} D_{\mu \lambda}\left[J_{\mu_{0} \lambda_{0}}-(1 / 2) K_{\mu_{0} \lambda_{0}}\right] \\
& +\sum_{\lambda_{0}}^{B_{0}} D_{j, \lambda} J_{\mu_{0} \lambda_{0}} \\
& +\sum_{j}\left[\sum _ { B _ { j } } \left(V_{\mu_{0} B_{j}}^{A_{0} B_{j}}+V_{\mu_{j} B_{0}}^{\left.A_{j} B_{0}\right)}\right.\right. \\
& \left.+D_{\mu \mu} \sum_{i}\left(J_{\mu_{0} \lambda_{j}}+J_{\mu_{j} \lambda_{0}}\right)\right] \\
F I_{\mu \nu}^{A A}= & (3 / 2) D_{\mu \nu} K_{\mu_{0} v_{0}}-(1 / 2) D_{\mu v} J_{\mu_{0} v_{0}} \\
F I_{\mu \nu}^{A B}= & t_{\mu_{0} v_{0}}-(1 / 2) D_{\mu v} J_{\mu_{0} v_{0}} ; \\
F K_{\mu \mu}(k)=\sum_{j}\left[2 \cos (k j) t_{\mu_{0} \mu_{j}}\right. & \left.\quad-\cos (k j) D_{\mu \mu}^{+}(j) J_{\mu_{0} \mu_{j}}\right]
\end{aligned}
$$




$$
\begin{aligned}
&{ }^{\mathrm{R}} F K_{\mu v}(k)=\sum_{j}[ \cos (k j)\left(t_{\mu_{0} v_{j}}+t_{\mu_{j} v_{0}}\right) \\
&-(1 / 2) \cos (k j)\left(D_{\mu v}^{+}(j) J_{\mu_{0} v_{j}}\right. \\
&\left.\left.+D_{v \mu}^{+}(j) J_{\mu_{j} v_{0}}\right)\right]
\end{aligned}
$$

$$
\begin{aligned}
{ }^{\mathrm{I}} F K_{\mu v}(k)=\sum_{j}\left[\sin (k j)\left(t_{\mu_{0} v_{j}}-t_{\mu_{j} v_{0}}\right)\right. \\
-(1 / 2) \sin (k j)\left[D_{\mu v}^{-}(j) J_{\mu_{0} v_{j}}\right. \\
\left.\left.\left.-D_{v \mu}^{-}(j) J_{\mu_{j} v_{0}}\right)\right]\right] .
\end{aligned}
$$

$H_{\mu \mu}$ in (15) corresponds to the atomic one-electron part (core Hamiltonian) of the HF operator, $V_{\mu_{0} B_{0}}^{A_{0} B_{0}}$ is the electron-core attraction between the $\mu^{\prime}$ th $\mathrm{AO}$ at atom $\mathrm{A}$ and the core of the $B^{\prime}$ th site of the reference cell. The first and second summations represent the one-center and two-center electron-electron repulsions within the reference cell $(j=0) . J_{\mu_{0} i_{0}}$ stands for the Coulomb interaction between the charge distributions $\left\langle\chi_{\mu}\left(r_{A}\right) \chi_{\mu}\left(r_{A}\right)\right|$ and $\left|\chi_{\lambda}\left(r_{A}\right) \chi_{\lambda}\left(r_{A}\right)\right\rangle$ while $K_{\mu_{0} \lambda_{0}}$ is the associated (one-center) exchange element. The first expression of the $j$-dependent sum is the electron-core attraction between $\chi_{\mu}\left(r_{A}\right)$ in the reference cell and the $B^{\prime}$ th core in the $j^{\prime}$ th cell. The last elements in (15) define the $k$-independent two-electron repulsions of the intercell-type. $t_{\mu_{0} v_{0}}$ in (17) is the one-electron hopping integral (i.e. kinetic energy operator) between the AO's $\chi_{\mu}\left(r_{A}\right)$ and $\chi_{v}\left(r_{B}\right)$ of the reference cell. The $k$-dependent matrix elements are summarized in (18) to (20). The diagonal of $F(k)$ contains the $\cos (k j)$-modulated hopping integrals $t_{\mu_{0} \mu_{j}}$ and $t_{\mu_{j} \mu_{0}}$, respectively, which fall off exponentially. Previous computational experience in the class of the metallomacrocycles has shown that these "covalent" integrals drop down to zero for interdeck separations exceeding ca. 3.8-4.0 $\AA$ [29]. The second elements in (18) are formally integrals of the Coulomb-type $\left(J_{\mu_{0} \mu_{j}}\right)$ but must be traced back to the antisymmetry of the HF determinant. These integrals are the "exchange counterpart" of the Coulomb repulsion $\left\langle\chi_{\mu}\left(r_{A}\right) \chi_{\mu}\left(r_{A}-j\right)\right.$ and $\left|\chi_{\mu}\left(r_{A}\right) \chi_{\mu}\left(r_{A}-j\right)\right\rangle$ which is of course set equal to zero in the ZDO framework. The $J_{\mu_{0} \mu_{j}}$ integrals in (18) fall off as $1 / j$ and the decay of the $k$-dependent exchange is therefore determined by the Fouriertransformed charge-density-bond-order matrices of (9). Similar combinations of hopping integrals and two-electron interactions via the nonlocal exchange are found in the real and imaginary parts of the off- diagonal elements of the mean-field operator ((19) and (20), respectively). The specific parametrization of the various interaction elements in the aforementioned equations is not important for the subsequent discussion. A detailed description of the approximations used in the adopted SCF HF INDO CO scheme is reported in [30].

The exchange contribution $E_{\mathrm{ex}}$ to the total energy of the polymer, $E_{\mathrm{tot}}$, is given by

$$
\begin{aligned}
E_{\mathrm{ex}}= & -(1 / 4) \sum_{A_{0}} \sum_{B_{0}} \sum_{\mu}^{A_{0}} \sum_{v}^{B_{0}} D_{\mu v}^{2} J_{\mu_{0} v_{0}} \\
& -(1 / 4) \sum_{j} \sum_{A_{0}} \sum_{B_{j}} \sum_{\mu}^{A_{0}} \sum_{v}^{B_{j}}\left[D_{\mu v}^{+}(j)\right]^{2} J_{\mu_{0} v_{j}},
\end{aligned}
$$

where the first term stands for the electronic exchange within the reference cell and the second one for the Fermi correlation between AO's that are $j$ unit cells apart. The total energy of the solid is symbolically written in the form of (22). $E_{\text {tot }}$ is given by the sum of the hopping energies $\left(E_{\mathrm{t}}\right)$, electron-electron $\left(E_{\mathrm{c}}\right)$ and core-core repulsions $\left(E_{\mathrm{cc}}\right)$, electron-core attraction $\left(E_{\mathrm{v}}\right)$ as well as the aforementioned $E_{\text {ex }}$ contribution:

$$
E_{\mathrm{tot}}=E_{\mathrm{t}}+E_{\mathrm{c}}+E_{\mathrm{cc}}+E_{\mathrm{v}}+E_{\mathrm{ex}} .
$$

All intercell interaction energies (with exception of the exchange) which enter the rhs. of (22) are independent of the matrix elements $D_{\mu \nu}^{ \pm}(j)$. The relevant formulas for the evaluation of $E_{\mathrm{tot}}$ are defined in [30].

The specific dispersion ( $k$-dependence) of a HF band is determined by the relative weight of the $\cos (k j)$-modulated hopping integrals $(2 \cos (k j)-$ $t_{\mu_{0} \mu_{j}}$ ) and the exchange energy contribution $\cos (k j) D_{\mu \mu}^{+}(j) J_{\mu_{0} \mu_{j}}$, respectively (diagonal elements of the HF operator). The one-electron part exceeds the latter sum in the case of strong covalent interactions between adjacent unit cells. Then the $\mathrm{HF}$ dispersions can be described in form of an idealized tight-binding curve which is (exclusively) determined by the nearest neighboring resonance integral(s) $t_{\mu_{0} \mu_{j}}(j=1)$. The restriction to $j=1$ is of course a direct consequence of the $\exp (-j)$ decay of the kietic energy integrals.

Tight-binding regime:

$$
\begin{aligned}
& \left|t_{\mu_{0} \mu_{j}}\right| \gg\left|D_{\mu \mu}^{+}(j) J_{\mu_{0} \mu_{j}}\right|, \\
& F K_{\mu \mu}(k) \approx 2 \cos (k j) t_{\mu_{0} \mu_{j}} .
\end{aligned}
$$


The $j=1$ approximation for the one-electron part of the crystal Hamiltonian is not longer valid in $1 \mathrm{D}$ (or even more in 2D and 3D) systems with small unit cell dimensions leading to short internuclear contacts between atomic centers that belong to different stacking units.

Weak intercell interactions (e.g., cluster structures, Van der Waals systems, donor-acceptor stacks, "active fragments" separated by rigid site groups, etc.) allow for a strong reduction of the hopping elements while the detraction of the exchange energy is less pronounced. The physical counterpart of the tight-binding regime (see (23) and (24)) is defined in (25) and (26), respectively. The analytic structure of a HF dispersion is now controlled by the electronic exchange.

Exchange-controlled regime:

$$
\begin{aligned}
& \left|t_{\mu_{0} \mu_{j}}\right| \ll\left|D_{\mu \mu}^{+}(j) J_{\mu_{0} \mu_{j}}\right|, \\
& F K_{\mu \mu}(k) \approx-\sum_{j} \cos (k j) D_{\mu \mu}^{+}(j) J_{\mu_{0} \mu_{j}} .
\end{aligned}
$$

Whether or not exchange contributions beyond $j=1$ are of significance in the expansion of (26) depends on the decay properties of $D_{\mu \mu}^{ \pm}(j) J_{\mu_{0} \mu_{j}}$. If we make use of the rough approximation to measure the $j$-variation of $D_{\mu \mu}^{ \pm}(j)$ by a constant $\varphi$ (with $\varphi<1$, see (27)),

$$
\varphi=\left|D_{\mu \mu}^{ \pm}(j+1)\right| /\left|D_{\mu \mu}^{ \pm}(j)\right|,
$$

then it is possible to express the asymptotic behaviour of the electronic exchange by the function $\varphi j^{-1}$. Detailed ab initio CO studies of simple 1D chains have shown that the magnitude of $\varphi$ depends critically on the interaction type $(\sigma, \pi$ or $\delta$ coupling), the stacking pattern of the polymer as well as the spatial extension of the atomic orbital wave function [7]. The fall-off parameter $\varphi j^{-1}$ determines the importance of $\cos (k j)$-modulated exchange contributions to the mean-field potential beyond $j=1$ (i.e., $\cos (2 k), \cos (3 k), \cos (4 k), \ldots$, etc.). The $\varepsilon(k)$ dispersion of an idealized tightbinding band (derived in the nearest neighbor's approximation) is perturbed in proportion to the influence of the long-range terms of the electronic exchange. The elements beyond $j=1$ lead to nonlinearities in $\varepsilon(k)$ diagrams due to the superposition of higher-order Fourier components $\cos (k), \cos (2 k)$, $\cos (3 k)$, etc. The second important consequence of significant exchange matrix elements is of course a broadening of the HF bands. The exchange-induced contributions to the HF operator are preponderantly restricted to $\varepsilon(k)$ modifications in the filled oneparticle space; this differs from the influence of the kinetic hopping integrals determining the widths of dispersion curves both in the filled and empty Fermi-seas.

To demonstrate this divergent behaviour between the kinetic energy contribution, on one side, and the Fermi correlation, on the other, we assume that a suitable unitary transformation of the Fock operator allows for a representation that diagonalizes the density matrix (occupation number representation). The $D_{a a}$ elements are either 2 (filled bands) or 0 (empty bands). The (diagonal) elements of the HF operator in this new basis are defined by

$$
\begin{aligned}
F I_{a a}= & H_{a a}+\sum_{B_{0}} V_{a B_{0}}^{B_{B_{0}}}+\sum_{b} D_{b b}\left[J_{a b}-(1 / 2) K_{a b}\right] \\
+ & +\sum_{j}\left[\sum_{B_{j}}\left(V_{a B_{j}}^{* B_{j}}+V_{a B-j}^{B-j}\right)\right. \\
& \left.\quad+D_{a a} \sum_{b}\left(J_{a b}^{*}+J_{b a}\right)\right], \\
F K_{a a}(k)= & \sum_{j}\left[\cos (k j)\left(t_{a a}^{*}+t_{a a}\right)\right. \\
& \left.-(1 / 2) \cos (k j) D_{a a}(j)\left(J_{a a}^{*}+J_{a a}\right)\right] ; \\
D_{a a}= & \begin{cases}2 & \text { filled bands } \\
0 & \text { empty bands } .\end{cases}
\end{aligned}
$$

Equation (29) shows immediately that the cos $(k j)$ dependence of the "classical" tight-binding elements is not restricted to the filled HF bands but spans the whole one-particle space. The $D_{a a}$ elements, on the other side, limit exchange-dependent phenomena (i.e., broadening of the bands, nonlinearities) to the filled bands of the infinite system.

In the following we want to assume that HF solutions according to (28) and (29), respectively, have been derived for a given array of neighbors $(j)$. Changes in the band structure properties due to alterations in the $j$-grid can be monitored by using first-order perturbation theory. For this purpose we abbreviate the row-vectors of the AO's by $\chi=\left(\chi_{1}\right.$, $\left.\gamma_{2}, \ldots\right)$ while occupied and empty canonical HF microstates derived for a preselected $j$-approximation are symbolized by $\psi_{\text {occ }}(k)_{j}$ and $\psi_{\text {vir }}(k)_{j}$. The lower $j$-index has been adopted to label the $j$ dependence of the Bloch orbitals. Then we can write

$$
\begin{aligned}
& \psi_{\text {occ }}(k)_{j}=\% \cdot c_{\text {occ }}(k)_{j}, \\
& \psi_{\text {vir }}(k)_{j}=\% \cdot c_{\text {vir }}(k)_{j},
\end{aligned}
$$




$$
\begin{aligned}
\psi(k)_{j} & =\left(\psi_{\mathrm{occ}}(k)_{j} \mid \psi_{\mathrm{vir}}(k)_{j}\right) \\
& =\chi \cdot\left(c_{\mathrm{occ}}(k)_{j} \mid c_{\mathrm{vir}}(k)_{j}\right)=\chi \cdot c(k)_{j} .
\end{aligned}
$$

$\psi(k)_{j}$ is the row-vector of all microstates with crystal momentum $k$ and $c(k)_{j}$ the unitary matrix which connects the canonical microstates with the AO basis functions. The modulation of these elements due to the $\exp (i k j)$ factor is trivial. Next we suppose that the row-vector of the filled levels derived in the $\tilde{j}$ 'th nearest neighbor's approximation $(\tilde{j} \neq j)$ can be expressed in the form

$$
\begin{aligned}
\psi_{\text {occ }}(k)_{j} & =\left(\psi_{\text {occ }}(k)_{j} \mid \psi_{\text {vir }}(k)_{j}\right)\left(I \mid M(k)^{-1}\right) \\
& =\left(\psi_{\text {occ }}(k)_{j} \mid \psi_{\text {vir }}(k)_{j}\right) c(k)_{j} .
\end{aligned}
$$

$I$ is the unit matrix of proper size (the dimension coincides with the number of occupied bands) and $M(k)$ corresponds to a mixing matrix between $k$ dependent states in the filled and empty Fermi-seas; $M_{a b}(k)$, e.g., is the mixing coefficient between the $a$ 'th occupied microstate and the $b$ 'th empty oneelectron function. The $\tilde{j}$ property is thereby expressed in terms of $j$-dependent parameters.

We can displayed the $\mathrm{HF}$ operator in the $\mathrm{CO}$ basis derived with $\tilde{j}$ neighbors in the lattice sum in terms of a nearly diagonal basis by means of crystal orbital coefficients $c_{\text {occ }}(k)_{j}$ and $c_{\text {vir }}(k)_{j}$, respectively, that correspond to a $\mathrm{CO}$ approach with $j$ adjacent stacking units considered in the lattice sum:

$$
\begin{aligned}
F(k)_{j}= & \left(c_{\mathrm{occ}}(k)_{j} \mid c_{\mathrm{vir}}(k)_{j}\right) \\
& \cdot F^{\mathrm{AO}}(k)_{j}\left(c_{\mathrm{occ}}(k)_{j} \mid c_{\mathrm{vir}}(k)_{j}\right) .
\end{aligned}
$$

In (35) we have used the symbol $\mathrm{AO}$ in order to indicate that the mean-field operator on the rhs has been defined in the AO basis. Equation (35) can be rearranged into the equation

$F(k)_{j}$

$=\left(\begin{array}{l|l}c_{\mathrm{occ}}(k)_{j} F^{\mathrm{AO}}(k)_{j} c_{\mathrm{occ}}(k)_{j} & c_{\mathrm{occ}}(k)_{j} F^{\mathrm{AO}}(k)_{j} c_{\mathrm{vir}}(k)_{j} \\ \hline c_{\mathrm{vir}}(k)_{j} F^{\mathrm{AO}}(k)_{j} c_{\mathrm{occ}}(k)_{j} & c_{\mathrm{vir}}(k)_{j} F^{\mathrm{AO}}(k)_{j} c_{\mathrm{vir}}(k)_{j}\end{array}\right)$

containing projections occ/occ, vir/vir and occ/vir.

In the limit of sufficiently large $j$ and $\tilde{j}$ dimensions it can be supposed that the occ/occ and vir/vir projections are in diagonal form; the crystal orbital coefficients derived for $j$ neighbors are pseudocanonical. Adopting first-order perturbation theory it is possible to express the mixing coefficients $M_{a b}(k)$ as

$$
M_{a b}(k)=F_{a b}(k)_{j} /\left(F_{a a}(k)_{j}-F_{b b}(k)_{j}\right),
$$

where $F_{a a}(k)_{j}$, etc. are the occ/occ, etc. projections of the mean-field operator in (36). Hence it is seen that exchange-dependent effects caused by modifications in the $\tilde{j}$-array are accompanied by a small intermixing between filled and empty HF microstates associated to $j$ nearest neighbors. Equations (35) to (37) are consistent with $D_{a a}$ parameters that are slightly smaller than 2 (derived for $j$ neighbors) and $D_{b b}$ elements close to zero $\left(D_{b b}>0, a \in\right.$ occ, $b \in \mathrm{vir})$. It is thus obvious that exchange energy effects are largely restricted to bands in the filled HF space. The approximate relations (28) to (37) are valid in the case of weak intercell interactions and $\mathrm{CO}$ microstates that depend not strongly on the value of the wave vector $k$.

The asymptotic influence of the exchange operator to the total energy expression corresponds to a $\varphi^{2} j^{-1}$ law $(\varphi<1.0)$ in contrast to the $\varphi j^{-1}$ decay encountered in the exchange matrix elements of the $\mathrm{HF}$ eigenvalue problem. Possible truncation errors are therefore of minor significance as long as $E_{\text {tot }}$ is considered; they are enlarged for the analysis of one-electron properties (i.e., band positions and band widths). It is thus a rather paradoxial situation that previous numerical studies were restricted to interpretations of total energies (with their weaker response to exchange-dependent phenomena) while band structure properties, which are more influenced by the Fermi correlation, were not analyzed as a function of the exchange asymptotics.

On the basis of the foregoing discussion it is obvious that $\varepsilon(k)$ relations of narrow-band materials as derived in the SCF HFCO approximation depend strongly on the asymptotic behaviour of the electronic exchange and thereby on the $j$-dependence of the $D_{\mu v}^{ \pm}(j)$ matrices defined in (9). Numerical errors in the integration of $D_{\mu v}^{ \pm}(j)$ should cause unphysical features in calculated band structure properties. It is essential to dispose of a numerical integration technique that allows for reliable extensions of the dimension of the lattice sum without enhancing the relative errors. It will be shown that attenuation factors $\tau_{j}$ are a simple and efficient tool to evaluate the Fourier coefficients of (9) [55]. A detailed analysis of the validity and the theoretical basis of this formalism will be given in a separate contribution [56].

Equation (9) shows that the $D_{\mu v}^{ \pm}(j)$ are the Fourier coefficients of a function $D_{\mu v}(k)$ which is not known analytically. The eigenvalue equations 
(6) render possible only a $D_{\mu v, k}$-grid at the preselected $k$-figures $k_{n}$ with $k_{n}=\pi n / N \quad(N$ is an integer and $n=0, \pm 1, \pm 2, \pm 3, \ldots$ ):

$$
D_{\mu v, k}=D_{\mu v}\left(k_{n}\right) \text {. }
$$

In order to find reliable approximations to $D_{\mu v}^{ \pm}(j)$ we make use of the method of trigonometric interpolation. The expansion coefficients of an interpolating polynomial are defined by

$$
\begin{aligned}
P_{\mu v}(k)= & \beta_{\mu v, 0}+\beta_{\mu v, 1} \exp (i k) \\
& +\beta_{\mu v, 2}(2 i k)+\ldots, \\
P\left(k_{n}\right)= & D_{\mu v, k}, \\
\beta_{\mu v, j}= & 2 \pi N^{-1} \sum_{n=0}^{(N-1)} D_{\mu v, k} \exp \left(-i j k_{n}\right) .
\end{aligned}
$$

The close correspondence between (41) and (9) suggests to adopt the $\beta_{\mu v, j}$ coefficients as approximations for the desired $D_{\mu v}(j)$ elements:

$$
D_{\mu v}(j)=\tau_{j} \cdot \beta_{\mu v, j} .
$$

In the case of standard interpolation schemes (e.g., Simpson's rule, discrete summation) $\tau_{j}$ is set equal to 1 irrespective of the value of $j$. It had been mentioned that this approximation leads to artificial periodicities in the intercell density matrix elements while the exact coefficients behave as

$$
\lim _{j \rightarrow \infty} D_{\mu v}(j)=0,
$$

which follows immediately from the convergence of the Fourier series

$$
D_{\mu v}(0)=\sum_{j=-\infty}^{+\infty} D_{\mu v}^{ \pm}(j) .
$$

The attenuation factors $\tau_{j}$ of (42) depend only on the interpolation scheme employed for $P(k)$ but are independent of the sequence of the $D_{\mu v, k}$. If linear or cubic spline functions are used to approximate $D_{\mu \nu}(k)$ between two subsequent $k$-points $k_{n}$ and $k_{n+1}[55,56]$, it can be shown that the $\tau_{j}$ are given by

$$
\tau_{j}=[(N / \pi j) \sin (\pi j / N)]^{2}
$$

and

$$
\begin{aligned}
\tau_{j}=3[\sin (\pi j / N) /(\pi j / N)]^{4} \\
\cdot\left[1+2 \cos ^{2}(\pi j / N)\right]^{-1},
\end{aligned}
$$

respectively.
Numerical studies have shown that the attenuation factors are close to 1 if the $k$-points exceed the $j$-array by a factor $>3$ [57]. $\tau_{j}$ is reduced to ca. 0.7 for mutual ratios of $2: 1$. The relative errors of integration procedures without the $\tau_{j}$-attenuation are then remarkably reinforced if $j$ is furthermore enlarged (relative to a fixed number of $k$-points). Such possible numerical inaccuracies have not been taken into account in several of the previously published $\mathrm{CO}$ studies. Therefore we believe that convergence properties sometimes have been pretended that must be traced back to computational round-off errors of the band structure calculations but were by no means physical manifestations of electronic structure effects.

\section{Simple Model Calculations}

In this section we investigate the $\varepsilon(k)$ dispersion of a single HF band as a function of the relative magnitude of the hopping matrix elements and the electronic exchange. For reasons of simplicity we employ a one-orbital model; the $k$-dependent elements of the HF operator are thus exclusively given by (18). With respect to $t_{\mu_{0 \mu_{j}}}$ we apply a strict tightbinding approximation, i.e. only the nearest neighbor's interaction is taken into account. Therefore relation (18) is simplified to

$$
\begin{aligned}
F K_{\mu \mu}(k)= & 2 \cos (k) t_{\mu_{0} \mu_{1}} \\
& -\sum_{j}\left[\cos (k j) D_{\mu \mu}^{+}(j) J_{\mu_{0} \mu_{j}}\right] .
\end{aligned}
$$

For the plain model we adopt a $D_{\mu \mu}^{+}(1) J_{\mu_{0} \mu_{1}}$ element of $0.5 \mathrm{eV}$. The $D_{\mu \mu}^{+}(j) J_{\mu_{0} \mu_{1}}$ figures beyond $j=1$ are evaluated by the interpolation

$$
\begin{aligned}
D_{\mu \mu}^{+}(j+1) J_{\mu_{0} \mu_{j+1}}= & \left|D_{\mu \mu}^{+}(j) J_{\mu_{0} \mu_{j}}\right| \\
& \cdot f \cdot( \pm 1)^{(j+1)} .
\end{aligned}
$$

The multiplicative factor $f$ with $f<0.7$ has the property of a decay amplitude. $f^{2}$ elements $<0.5$ are necessary to guarantee the convergence of the total energy of the model solid. $( \pm 1)^{(j+1)}$ has been included to simulate either alternating signs between adjacent $D_{\mu \mu}^{+}(j)$ elements or $D_{\mu \mu}^{+}(j)$ arrays that decay monotonously (see [58]). The two expansions with $(+1)^{(j+1)}$ and $(-1)^{(j+1)}$ give rise to dispersion curves that are connected by an inversion at $k=\pi / 2$; exchange-induced $\varepsilon(k)$ variations at the edge of the Brillouin zone for $(-1)^{(j+1)}$ cor- 
respond to modifications at the zone center for $(+1)^{(j+1)}$ and vice versa. We have considered $f$ factors of $0.25(\mathbf{A}), 0.45(\mathbf{B})$ and $0.65(\mathbf{C})$ which simulate different conditions of the decay of the electronic exchange. The subsequent examples correspond to an alternating decay potential controlled via $(-1)^{(j+1)}$.

Three representative $\varepsilon(k)$ diagrams for $\mathbf{A}$ are summarized in the Figs. $2(j=1), 3(j=2)$ and 4 $(j=4)$. The numbers in parentheses indicate the employed neighbor's approximation. The plot in Fig. 2 with $j=1$ shows the well-known idealized tight-binding relation. Additional elements $j=2$ and $j=4$ allow for a broadening of the dispersion curve and lead furthermore to a flattening of the

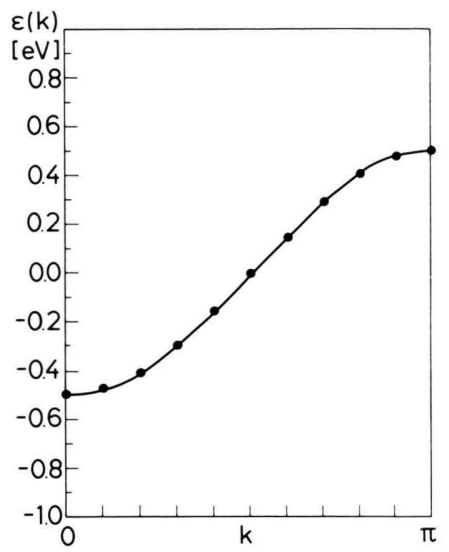

Fig. 2. Idealized tight-binding band associated to the exchange model A derived in the nearest neighbor's approximation $(j=1)$.
HF band in the neighborhood of the $\Gamma$-point but to enlarged energy gradients in the second half of the Brillouin zone.

These deformations of the $\varepsilon(k)$ curves are reinforced in example $\mathbf{B}$. The dispersions determined by the exchange potential of (47) are displayed in the Figs. $5(j=2), 6(j=3)$ and $7(j=4)$. The increasing $D_{\mu \mu}^{+}(j) J_{\mu_{0} \mu_{j}}$ elements for $j>1$ are responsible for the remarkable nonlinearities in the first half of the Brillouin zone. The negative slope (near the $\Gamma$-point) of the $\varepsilon(k)$ curve in Fig. 5 must be traced back to the $j=2$ term in (47) or (48), respectively. The increasing LR exchange contributions in $\mathbf{B}$ make the details of a given dispersion strongly dependent on the dimension employed for the lattice summation (e.g. effective mass parameters at certain $k$-points). The total width of the band is less influenced by the $j$-array.

The last series of $\varepsilon(k)$ diagrams (only exchange contributions) is given in the Figs. 8 to 11 . The diagrams are based on the decay potential $\mathbf{C}$ and correspond to $j=2,3,4$ and 5 , respectively. The inversion symmetry of an idealized tight-binding band at $k= \pm \pi / 2$ is completely perturbed. The $\varepsilon(k)$ curves show "oscillations" for small crystal momenta; increasing $\varepsilon(k)$ gradients, on the other side, are predicted for larger values of the wave vector $k$. A fine grid of $k$-points is essential for an accurate representation of the HF dispersions.

Figure 12 symbolizes the modifications in the density of states distribution, $N(E)$, as a function of increasing LR exchange terms. The $N(E)$ histogram at the extreme left (two identical $N(E)$ maxi-

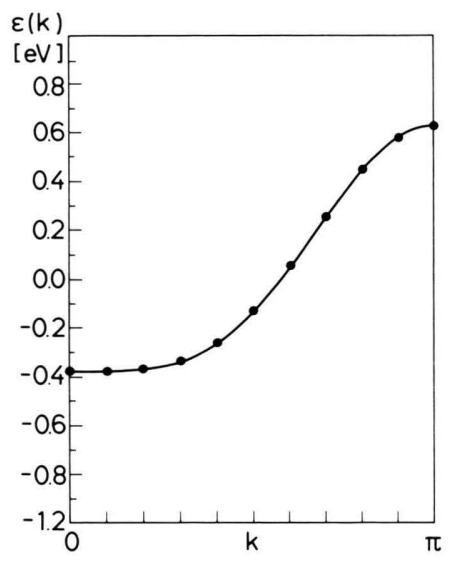

Fig. 3. Dispersion of the exchange model A with $j=2$.

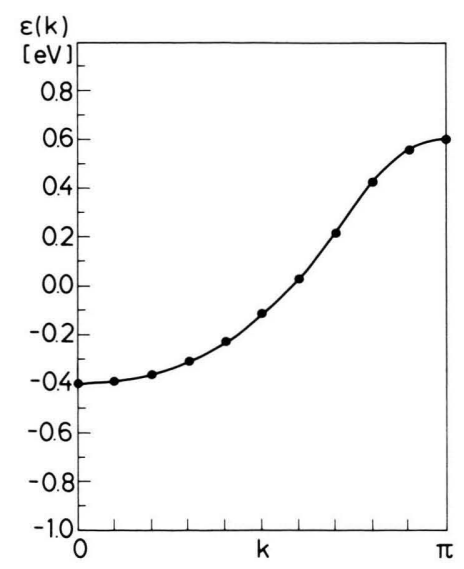

Fig. 4. Dispersion of the exchange model A with $j=4$.

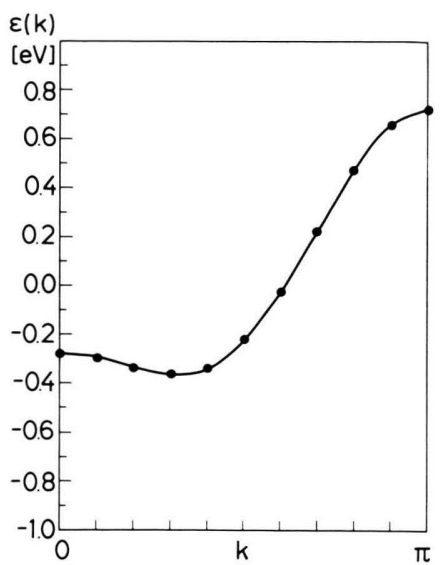

Fig. 5. Dispersion of the exchange model $\mathbf{B}$ with $j=2$. 
$\varepsilon(k)$

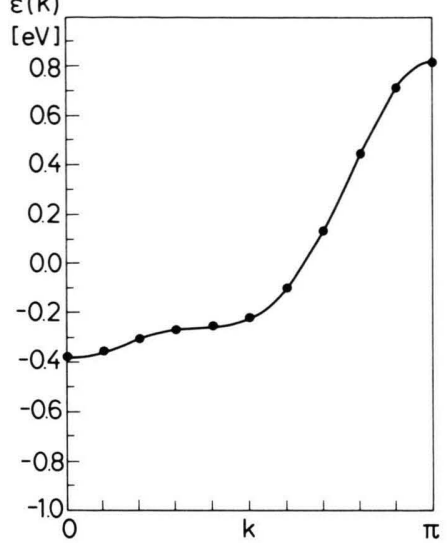

Fig. 6. Dispersion of the exchange model $\mathbf{B}$ with $j=3$.

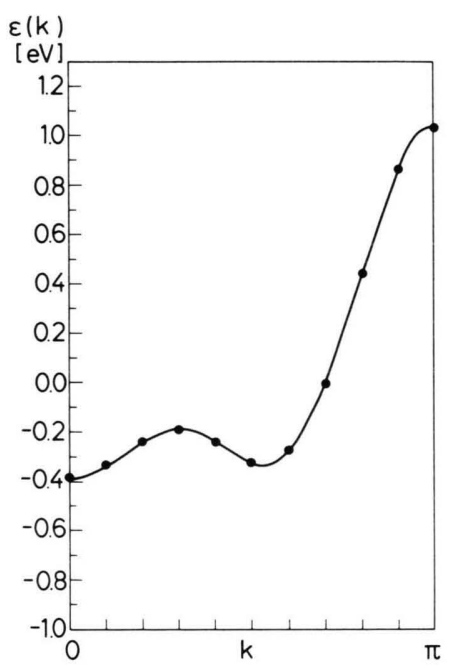

Fig. 9. Dispersion of the exchange model $\mathbf{C}$ with $j=3$.

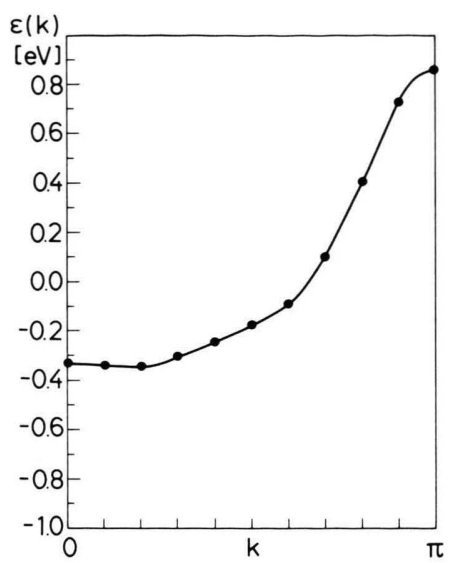

Fig. 7. Dispersion of the exchange model $\mathbf{B}$ with $j=4$.

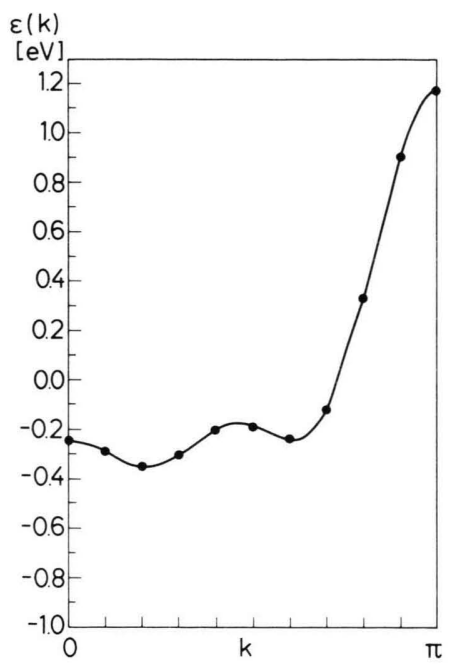

Fig. 10. Dispersion of the exchange model $\mathbf{C}$ with $j=4$.

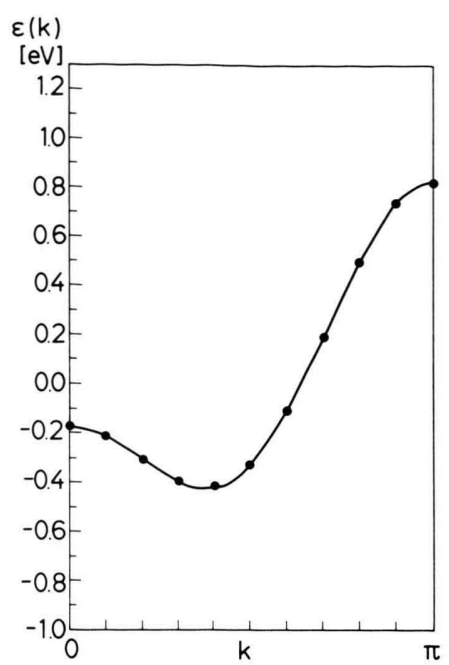

Fig. 8. Dispersion of the exchange model $\mathbf{C}$ with $j=2$.

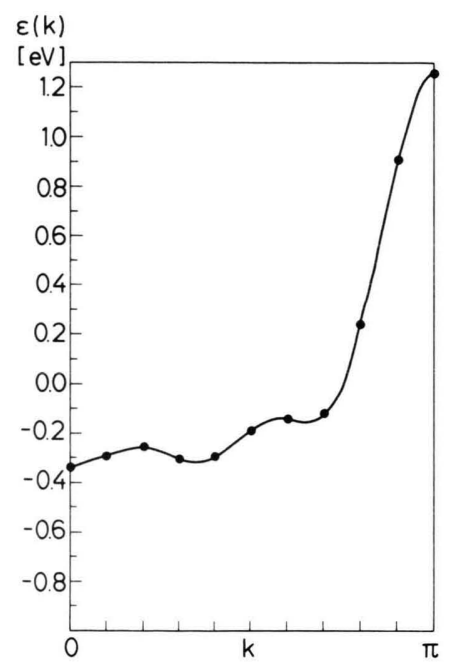

Fig. 11. Dispersion of the exchange model $\mathbf{C}$ with $j=5$. ma (singularities) associated to $k=0$ and $k= \pm \pi$ ) is typical for the nearest neighbor's approximation. The additional exchange contributions beyond $j=1$ enlarge the width of the DOS distribution. In addition they reduce the $N(E)$ peaks caused by the microstates at the top of the band but increase simultaneously the $k=0$ maxima.

In the dispersion curves of the Figs. 2 to 11 only the exchange-dependent contributions to the net HF dispersions have been considered as a function of the decay parameter $f$. In the subsequent figures $\varepsilon(k)$ relations are presented that contain the superposition of the two $k$-dependent terms entering the rhs. of (47). For this purpose we adopted exchange model $\mathbf{C}$ in the fifth nearest neighbor's approximation (see Figure 11). Net HF dispersions are displayed in the Figs. 13 and 14 which are based on this exchange potential and resonance integrals $t_{\mu_{0} \mu_{1}}$ typically found in narrow-band materials. The employed $t_{\mu_{0} \mu_{1}}$ elements are $-0.125 \mathrm{eV}$ and $-0.375 \mathrm{eV}$, respectively. The curves in Fig. 13 result from the superposition of resonance and exchange disper- 

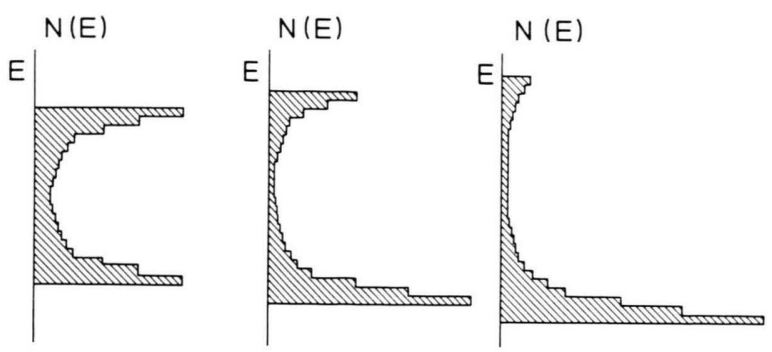

Fig. 12. Modification of the density of states distribution, $N(E)$, due to increasing long-range terms of the electronic exchange (from the lhs. of the figure to the rhs.). The first histogram corresponds to the $N(E)$ distribution derived for an idealized tight-binding relation in the nearest neighbor's approximation. The $N(E)$ maxima associated to $k= \pm \pi$ are reduced with an increasing strength of the exchange potential while the density of states maximum caused by the bottom of the band $(k=0)$ is raised. The schematic $N(E)$ histograms are valid for the dispersions which are plotted in the Figs. 2 to 11; alternating signs between adjacent $D_{\mu \nu}^{ \pm}(j)$ elements have been assumed. Intercell bond-order matrices that decay monotonously lead to an interchange of the two (inequivalent) $N(E)$ maxima determined by the DOS distributions at the top and bottom of the band.

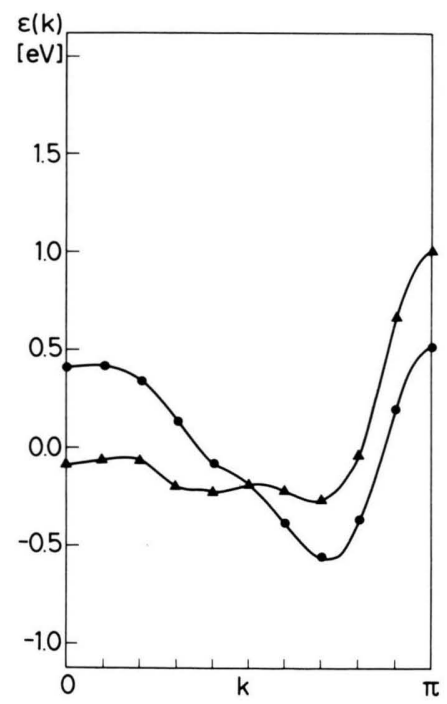

Fig. 13. Superposition of the $k$-dependent hopping and exchange matrix elements of the one-orbital model defined in (47). We have employed exchange potential $\mathbf{C}$ in the fifth nearest neighbor's approximation. The $\varepsilon(k)$ curve labelled by triangles corresponds to the "sum curve" of the adopted exchange model combined with a nearest-neighbor hopping matrix element $t_{\mu_{0} \mu_{1}}$ of $-0.125 \mathrm{eV}$. The $\varepsilon(k)$ relation marked by full circles is derived for a resonance integral of $-0.375 \mathrm{eV}$. The gradients of the exchange and one-electron contributions to (47) differ (exchange: positive gradient over the whole Brillouin zone, kinetic energy: negative gradient).

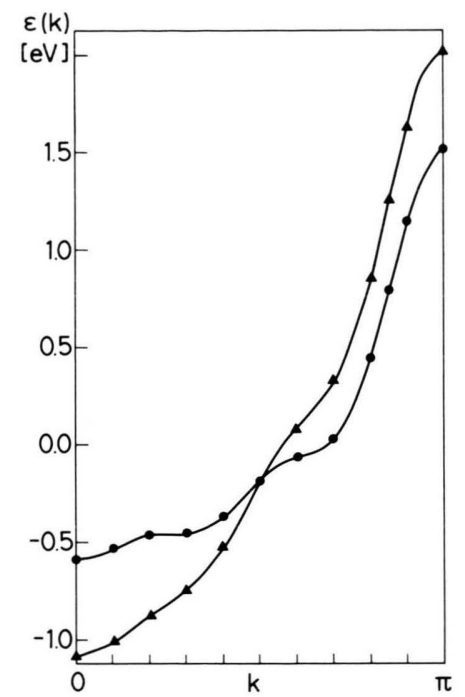

Fig. 14. Superposition of the $k$-dependent exchange and hopping matrix elements displayed in Fig. 13 but with positive gradients of both $k$-dependent contributions to the mean-field potential defined in (47).

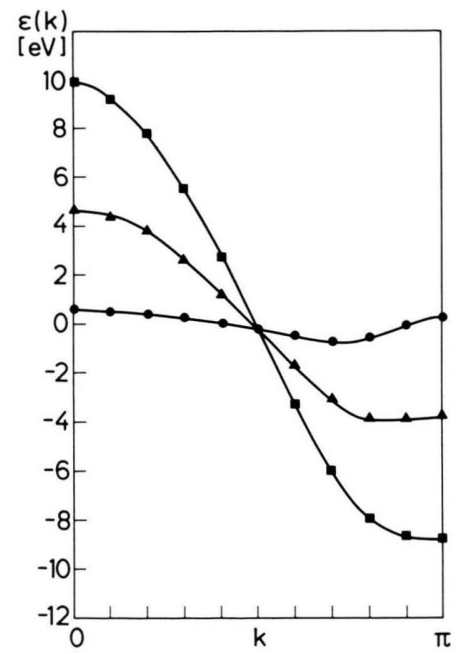

Fig. 15. Superposition of the $k$-dependent hopping and exchange matrix elements of the one-orbital model defined in (47). In analogy to the Figs. 13 and 14 the exchange potential $\mathbf{C}$ with $j=5$ has been used as common oneelectron potential. The $\bullet$ symbols correspond to a $t_{\mu_{0} \mu}$ element of $-0.5 \mathrm{eV}, \boldsymbol{\Delta}$ to $-2.5 \mathrm{eV}$ and to $-5.0 \mathrm{eV}$. A negative gradient in the dispersion of the kinetic hopping integrals has been employed. 


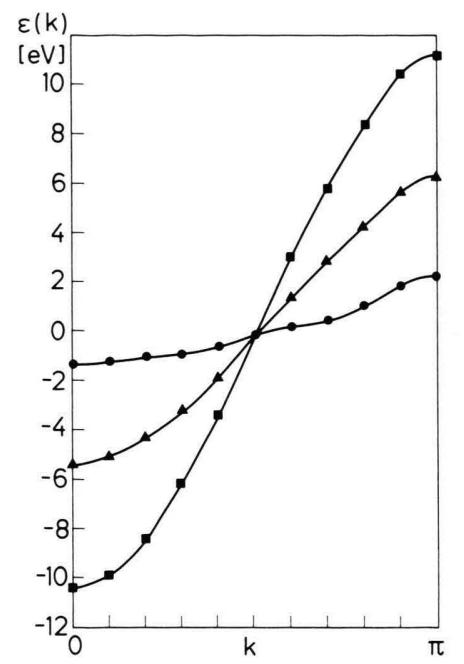

Fig. 16. Superposition of the $k$-dependent exchange and hopping matrix elements displayed in Fig. 15 but with resonance integrals defining a positive energy gradient.

sions of opposite slopes (exchange part: positive slope; one-electron integral: negative slope). Additive interference effects are schematized in Figure 14. The individual gradients are both positive.

Figure 13 shows impressively the strong perturbations of the $2 \cos (k) t_{\mu_{0} \mu_{1}}$ element due to the $k$ dependent two-electron potential. Small modifications in the magnitudes of the hopping and exchange contributions to the mean-field operator lead to significant changes in the shape of the $\varepsilon(k)$ curves. Possible deviations from an idealized tightbinding behaviour are reduced due to the conditions displayed in Figure 14. CO calculations based on a coarse grid of $k$-points may lead to the (incorrect) impression that these HF bands can be well described by a simple tight-binding relation.

Sum curves (exchange plus hopping contribution) for one-electron matrix elements $t_{\mu_{0} \mu_{1}}=-0.5,-2.5$ and $-5.0 \mathrm{eV}$ are shown in the Figs. 15 and 16. The first display corresponds once again to the combination of opposite gradients of the one- and two-electron potentials of (47). Figure 16 is the "broadband" counterpart of Figure 14. The $k$-dependent perturbations of the HF bands are partially suppressed due to the enlarged resonance integrals. The exchanged-induced nonlinearities are hidden in the two examples with $t_{\mu_{0} \mu_{1}}=-2.5$ and $-5.0 \mathrm{eV}$. The onset of the LR exchange terms appears in the $\varepsilon(k)$ curve with $t_{\mu_{0} \mu_{1}}=-0.5 \mathrm{eV}$. Once again it has to be mentioned that unreliable dispersion curves are derived in $\mathrm{CO}$ approaches with an imbalanced number of $k$-points.

The phenomena discussed in this paragraph are only observed in CO variants based on the SCF HF approximation where (nonlocal) exchange potentials are properly taken into account. They are of course not part of one-electron schemes of the WHor EH-type. In principle it is possible to detect these nonlinearities etc. also in tight-binding methods defined in the framework of the local density approximation (LDA) [59]. The employed $[\varrho(r)]^{1 / 3}$ potential (free-electron exchange), however, should diminish the onset of this two-electron control in the CO scheme.

\section{Computational Details}

In the $\mathrm{CO}$ calculations of the employed porphyrinato nickel(II) models $\mathbf{2}$ and $\mathbf{3}$, respectively, a grid of $10 \mathrm{k}$-points has been adopted. Lattice sum dimensions from $j=1$ (nearest neighbor's approximation) to $j=5$ were considered. The above described attenuation factors $\tau_{j}$ have been used for the numerical integration of the charge-density-bondorder matrices; we made use of (46) which is valid for cubic spline functions.

For reasons of computational simplicity we have restricted the tight-binding analysis to non-paramagnetic (unoxidized) states of the complex 1D materials; the HF-bands are thus either completely filled or empty. The severe numerical problems already encountered in partially oxidized chains with smaller stacking units have been mentioned in $[27,60,61]$. An accelerated Hartree damping algorithm of the charge-density-bond-order matrices has been used throughout the iterative SCF steps in order to prevent shell (band) swapping between the occupied and virtual one-particle levels [62]. SCF band states have been selected on the basis of oneelectron calculations of the WH-type. Several (band) occupation patterns of $\mathbf{2}$ and $\mathbf{3}$ were iterated up to a SCF energy criterion of $10^{-4} \mathrm{au}$. The results in the next section correspond to those mean-field solutions that are lowest in energy.

The experimental X-ray parameters of 2 [35] and 3 [36] have been used in the CO calculations. All band structure calculations were performed at eclipsed structure with a torsion of $0^{\circ}$ between direct neighbors. The experimental stacking pattern 
of $\mathbf{2}$ and $\mathbf{3}$, however, corresponds to a staggered $1 \mathrm{D}$ arrangement with an angle $\neq 0^{\circ}$. Model calculations have shown that the rotational barriers of $\mathbf{2}$ and $\mathbf{3}$ are very flat [50]; the relevant one-particle properties of the $\mathrm{Ni}(\mathrm{II})$ porphyrines are also not strongly changed due to a mutual intercell rotation. The accepted $0^{\circ}$ geometry warrants furthermore the conservation of maximum symmetries of the $\mathrm{CO}$ wave functions in $k$-space. A standard bond lengths of $1.1 \AA$ has been adopted for the $\mathrm{CH}$ bonds of 2 and $\mathbf{3}[63]$.

\section{Exchange-Influence on Band Structure Properties of 2 and 3}

In the subsequent $\mathrm{CO}$ calculations the labels $\mathbf{2}$ and $\mathbf{3}$ are used to abbreviate the weakly and highly puckered porphyrinato nickel(II) polymers. The common 1D skeleton, however, corresponds to the unsubstituted small-ring system 4. The total energies (normalized to one unit cell), $E_{\text {tot }}$, of $\mathbf{2}$ and $\mathbf{3}$ are shown in the Figs. 17 and 18 as a function of the

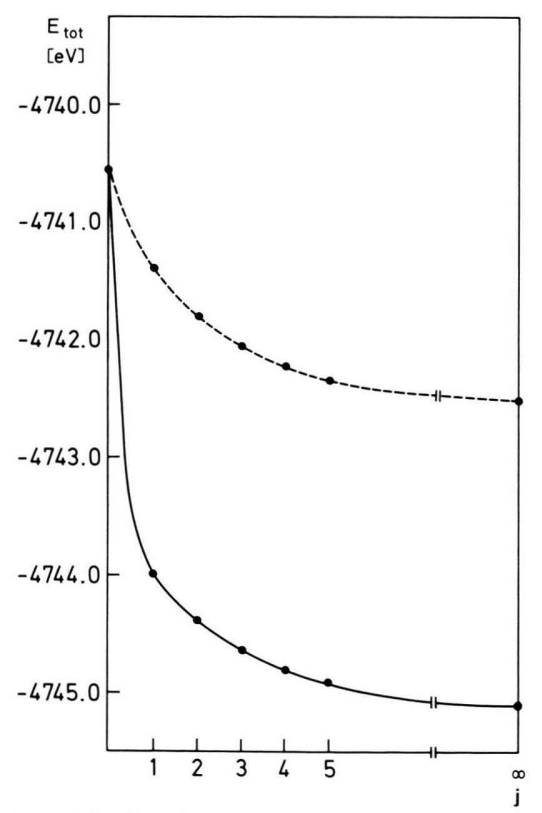

Fig. 17. Total energy $E_{\text {tot }}$ of $\mathbf{2}$ in the framework of the semiempirical SCF HF INDO CO scheme as a function of the number of adjacent unit cells considered in the lattice sum $(j=1$ to $j=5)$. $E_{\text {tot }}$ for $j=0$ corrresponds to the HF energy of the free $\mathrm{Ni}(\mathrm{P})$ molecule. The broken curve symbolizes $E_{\text {tot }}$ where the nearest neighboring hopping interaction has been removed $(-2.581 \mathrm{eV})$. This curve describes therefore the $j$-dependence of the electronic exchange. lattice sum dimension $j$. Additionally net HF energies of the discrete molecules $\mathbf{2}$ and $\mathbf{3}$ (i.e. $j=0$ ) are included in the display. The $j$-dependence of $E_{\text {tot }}$ indicates the superposition of the aforementioned intercell coupling mechanisms: the fast decay due to the short-range kinetic hopping integrals as well as the exchange potential which converges slower. The analytic difference between the $k$-dependent oneand two-electron parts of the crystal Hamiltonian is quantified in Table 1 containing intercell energies $E_{\text {inter }}$ which have been divided into $E_{\mathrm{t}}$ (resonance interaction), $E_{\text {cou }}$ (classical electrostatic interaction energy; sum of electron-electron and core-core repulsions and electron-core attraction, $E_{\mathrm{cou}}=E_{\mathrm{c}}+$ $\left.E_{\mathrm{cc}}+E_{\mathrm{v}}\right)$ and the exchange potential $E_{\mathrm{ex}}$. We have summarized only elements derived in the fifth nearest neighbor's approximation (intercell potentials from the nearest to the fifth nearest neighboring moiety). The coupling energies evaluated in $\mathrm{CO}$ calculations with $j=1$ to $j=4$ are nearly identical with the results of Tab. 1; the individual contributions to $E_{\text {tot }}$ are highly additive and quite independent from the employed $j$-array [22].

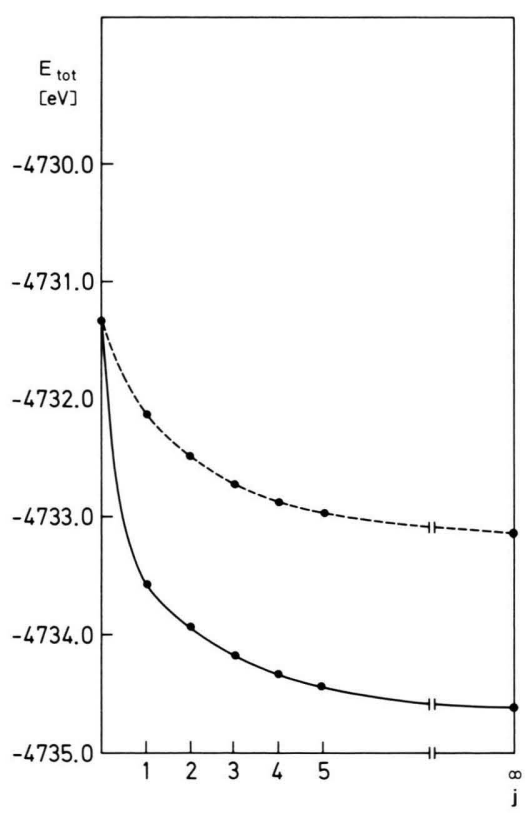

Fig. 18. Total energy $E_{\text {tot }}$ of $\mathbf{3}$ in the framework of the semiempirical SCF HF INDO CO scheme as a function of the number of adjacent unit cells considered in the lattice sum $(j=1$ to $j=5)$; see legend Fig. 17. The removed hopping interaction in the broken curve corresponds to $-1.447 \mathrm{eV}$. 
The classical Coulomb energies of the two uncharged systems are of course negligibly small in comparison to $E_{\mathrm{t}}$ and $E_{\mathrm{ex}}$. The interaction energy with the nearest neighboring units $j= \pm 1$ is prevailingly determined by the one-electron part of the HF operator. The hopping matrix elements beyond the direct neighbors, however, drop down to zero in both polymers. The $0.322 \AA$ elongation by going from 2 to 3 is accompanied by a $44 \%$ reduction of the kinetic intercell contribution. The magnitude of the exchange potential, on the other side, is not significantly modified due to the increasing interdeck separation. The $E_{\mathrm{ex}}$ figures of $\mathbf{2}$ and $\mathbf{3}$ are roughly comparable.

Table 1. Fragmentation of the net intercell energies $E_{\text {inter }}$ of $\mathbf{2}$ and $\mathbf{3}$ into resonance $\left(E_{\mathrm{v}}\right)$, classical electrostatic $\left(E_{\mathrm{cou}}\right)$ and exchange $\left(E_{\text {ex }}\right)$ contributions according to INDO CO calculations derived in the fifth neighbor's approximation. $E_{\text {inter }}$ is divided into interaction energies between the reference cell and the first, second, etc. adjacent neighbor $(j= \pm 1, \pm 2$, etc. $)$. All values in $\mathrm{eV}$.

\begin{tabular}{lccccc}
\hline Model & $j$ & \multicolumn{1}{l}{$E_{\mathrm{t}}$} & \multicolumn{1}{l}{$E_{\text {cou }}$} & $E_{\text {ex }}$ & $E_{\text {inter }}$ \\
\hline $\mathbf{2}$ & 1 & -2.581 & 0.022 & -0.887 & -3.446 \\
& 2 & -0.004 & -0.031 & -0.365 & -0.400 \\
& 3 & - & -0.013 & -0.247 & -0.260 \\
& 4 & - & -0.007 & -0.167 & -0.174 \\
& 5 & - & -0.004 & -0.113 & -0.117 \\
$\mathbf{3}$ & 1 & -1.447 & -0.019 & -0.770 & -2.236 \\
& 2 & -0.001 & -0.026 & -0.336 & -0.363 \\
& 3 & - & -0.011 & -0.227 & -0.238 \\
& 4 & - & -0.005 & -0.153 & -0.158 \\
& 5 & - & -0.003 & -0.104 & -0.107 \\
\hline
\end{tabular}

Artificial periodicities or local $E_{\text {inter }}$ maxima beyond $j= \pm 1$ are not found in the theoretical results summarized in the Figs. 17 and 18 or Table 1, respectively. The employed computational conditions guarantee convergent lattice sums in both model stacks 2 and 3 . The intercell potentials of the interaction between the reference cell and the fifth neighbouring molecule are reduced to figures smaller than $3 \mathrm{kcal}$. These interaction elements exceed the adopted SCF energy criterion by only one order of magnitude.

Atomic net charges $q_{\mathrm{A}}$ [64] of $\mathbf{2}$ and $\mathbf{3}$ are summarized in Table 2. The $q_{\mathrm{A}}$ numbers have been derived in five separate $\mathrm{CO}$ calculations spanning $j$-arrays from 1 to 5 . The charge distribution (determined by the intercell bond-order matrix of (8)) is already stabilized for $\mathrm{CO}$ schemes based on the third nearest neighbor's approximation. But even the $q_{\mathrm{A}}$ modifications observed in the step from $j=1$ to $j=2$ are smaller than ca. $0.5 \%$.

Important band structure properties of $\mathbf{2}$ and $\mathbf{3}$ as a function of the lattice sum dimension $j$ are given in Table 3. We have collected the $\varepsilon(k)$ values of the conduction $(c)$ and valence $(v)$ bands of the unoxidized materials at the marginal $k$-points $\Gamma$ and $X$. The corresponding $\varepsilon(k)$ figures of $(v-1)$ are also given in the table. $\Delta \varepsilon$ is the width of the HF dispersion and $\Delta E_{\mathrm{G}}$ the forbidden band gap. The numerical results in Table 3 show the marked $j$ dependence of certain one-electron properties in contrast to the $j$-independent $q_{\mathrm{A}}$ spectrum of Table 2. The exchange influence on band structure

Table 2. Atomic net charges $q_{\mathrm{A}}$ of the porphyrinato nickel(II) models $\mathbf{2}$ and $\mathbf{3}$ as a function of the lattice sum dimension $j$ according to the semiempirical SCF INDO CO formalism. The atomic numbering scheme is given in Figure 1.

\begin{tabular}{|c|c|c|c|c|c|c|}
\hline Model & Atom & $j=1$ & $j=2$ & $j=3$ & $j=4$ & $j=5$ \\
\hline 2 & $\begin{array}{l}\mathrm{Ni} \\
\mathrm{N} \\
\mathrm{C}_{1}=\mathrm{C}_{4} \\
\mathrm{C}_{2}=\mathrm{C}_{3} \\
\mathrm{C}_{5} \\
\mathrm{H}_{2}=\mathrm{H}_{3} \\
\mathrm{H}_{5}\end{array}$ & $\begin{array}{r}1.2270 \\
-0.6520 \\
0.3238 \\
-0.1733 \\
-0.3313 \\
0.1284 \\
0.1187\end{array}$ & $\begin{array}{r}1.2304 \\
-0.6472 \\
0.3251 \\
-0.1759 \\
-0.3310 \\
0.1273 \\
0.1177\end{array}$ & $\begin{array}{r}1.2313 \\
-0.6458 \\
0.3253 \\
-0.1767 \\
-0.3310 \\
0.1271 \\
0.1175\end{array}$ & $\begin{array}{r}1.2316 \\
-0.6453 \\
0.3254 \\
-0.1770 \\
-0.3310 \\
0.1270 \\
0.1174\end{array}$ & $\begin{array}{r}1.2317 \\
-0.6450 \\
0.3255 \\
-0.1771 \\
-0.3310 \\
0.1270 \\
0.1174\end{array}$ \\
\hline 3 & $\begin{array}{l}\mathrm{Ni} \\
\mathrm{N}_{1}=\mathrm{C}_{4} \\
\mathrm{C}_{1}=\mathrm{C}_{3} \\
\mathrm{C}_{2}=\mathrm{C}_{3} \\
\mathrm{C}_{5} \\
\mathrm{H}_{2}=\mathrm{H}_{3} \\
\mathrm{H}_{5}\end{array}$ & $\begin{array}{r}1.2379 \\
-0.6609 \\
0.3249 \\
-0.1774 \\
-0.3202 \\
0.1307 \\
0.1154\end{array}$ & $\begin{array}{r}1.2394 \\
-0.6592 \\
0.3254 \\
-0.1786 \\
-0.3200 \\
0.1302 \\
0.1153\end{array}$ & $\begin{array}{r}1.2398 \\
-0.6587 \\
0.3256 \\
-0.1790 \\
-0.3200 \\
0.1301 \\
0.1153\end{array}$ & $\begin{array}{r}1.2399 \\
-0.6585 \\
0.3256 \\
-0.1791 \\
-0.3200 \\
0.1301 \\
0.1153\end{array}$ & $\begin{array}{r}1.2400 \\
-0.6584 \\
0.3256 \\
-0.1792 \\
-0.3200 \\
0.1301 \\
0.1153\end{array}$ \\
\hline
\end{tabular}


Table 3. Band structure properties of $\mathbf{2}$ and $\mathbf{3}$ as a function of the lattice sum dimension $j$. We have summarized the band energies of the conduction $(c)$ and valence $(v)$ bands at the marginal $k$-points $k=0$ and $k=\pi ;(v-1)$ is the band below the valence band. $\Delta \varepsilon$ corresponds to the width of the $\mathrm{HF}$ dispersions. $\Delta E_{\mathrm{G}}$ symbolizes the forbidden band gap of the unoxidized non-paramagnetic materials. All values in $\mathrm{eV}$.

\begin{tabular}{|c|c|c|c|c|c|c|}
\hline Model & & $j=1$ & $j=2$ & $j=3$ & $j=4$ & $j=5$ \\
\hline 2 & $\begin{array}{l}\varepsilon_{c}(0) \\
\varepsilon_{c}(\pi) \\
\Delta \varepsilon_{c} \\
\varepsilon_{v}(0) \\
\varepsilon_{v}(\pi) \\
\Delta \varepsilon_{v} \\
\varepsilon_{(v-1)}(0) \\
\varepsilon_{(v-1)}(\pi) \\
\left.\Delta \varepsilon_{(v}-1\right) \\
\Delta E_{\mathrm{G}}\end{array}$ & $\begin{array}{r}-2.125 \\
-2.893 \\
0.788 \\
-6.352 \\
-7.365 \\
1.013 \\
-8.145 \\
-9.044 \\
0.900 \\
3.44\end{array}$ & $\begin{array}{r}-1.985 \\
-2.772 \\
0.785 \\
-6.082 \\
-7.096 \\
1.015 \\
-7.865 \\
-8.745 \\
0.902 \\
3.31\end{array}$ & $\begin{array}{r}-1.931 \\
-2.708 \\
0.777 \\
-6.121 \\
-6.936 \\
0.926 \\
-7.906 \\
-8.575 \\
0.885 \\
3.41\end{array}$ & $\begin{array}{r}-1.896 \\
-2.674 \\
0.778 \\
-6.012 \\
-6.827 \\
1.075 \\
-7.795 \\
-8.462 \\
1.001 \\
3.34\end{array}$ & $\begin{array}{r}-1.880 \\
-2.655 \\
0.774 \\
-6.056 \\
-6.747 \\
1.080 \\
-7.840 \\
-8.380 \\
0.992 \\
3.39\end{array}$ \\
\hline 3 & $\begin{array}{l}\varepsilon_{c}(0) \\
\varepsilon_{c}(\pi) \\
\Delta \varepsilon_{c} \\
\varepsilon_{v}(0) \\
\varepsilon_{v}(\pi) \\
\Delta \varepsilon_{v} \\
\varepsilon_{(v-1)}(0) \\
\varepsilon_{(v-1)}(\pi) \\
\Delta \varepsilon_{(v-1)} \\
\Delta E_{\mathrm{G}}\end{array}$ & $\begin{array}{r}-2.583 \\
-2.650 \\
0.074 \\
-7.015 \\
-6.831 \\
0.193 \\
-8.388 \\
-8.589 \\
0.201 \\
4.17\end{array}$ & $\begin{array}{r}-2.492 \\
-2.559 \\
0.068 \\
-6.806 \\
-6.622 \\
0.283 \\
-8.170 \\
-8.367 \\
0.403 \\
4.06\end{array}$ & $\begin{array}{r}-2.457 \\
-2.518 \\
0.061 \\
-6.860 \\
-6.491 \\
0.395 \\
-8.226 \\
-8.232 \\
0.374 \\
3.97\end{array}$ & $\begin{array}{r}-2.435 \\
-2.496 \\
0.062 \\
-6.769 \\
-6.399 \\
0.480 \\
-8.133 \\
-8.139 \\
0.476 \\
3.90\end{array}$ & $\begin{array}{r}-2.424 \\
-2.483 \\
0.061 \\
-6.814 \\
-6.330 \\
0.509 \\
-8.179 \\
-8.068 \\
0.499 \\
3.85\end{array}$ \\
\hline
\end{tabular}

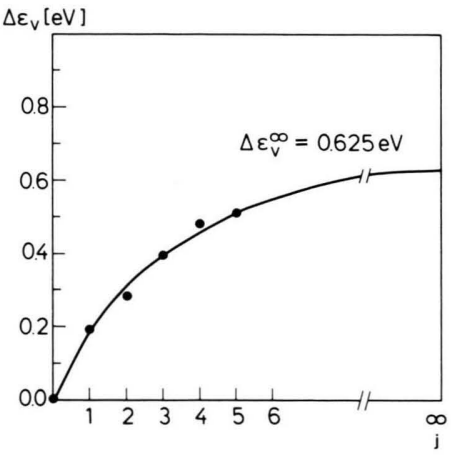

Fig. 19. Width of the valence band $(v), \Delta \varepsilon_{v}$, of $\mathbf{3}$ as a function of the lattice sum dimension employed in the semiempirical SCF HF INDO CO approximation. $\Delta \varepsilon_{v}^{\infty}$ is the "exact" HF band width corresponding to an extrapolation to $N \rightarrow \infty$.

parameters is extremely important in the highly puckered porphyrinato $\mathrm{Ni}$ (II) model 3 but is reduced in $\mathbf{2}$ with the enlarged hopping matrix elements. The valence band of $\mathbf{3}$ is broadened from $0.19 \mathrm{eV}(j=1)$ to $0.51 \mathrm{eV}(j=5)$. A similar $\Delta \varepsilon$ interval is predicted for the second highest filled band $(v-1)(0.20 \mathrm{eV} \rightarrow 0.50 \mathrm{eV})$. Both dispersion curves are formed by $\pi$ orbitals of the macrocyclic ligand frames [25, 26, 48, 49]. An approximate graphic extrapolation of $\Delta \varepsilon_{v}$ to $N \rightarrow \infty$ is displayed in Figure 19. $\Delta \varepsilon_{v}^{\infty}$ amounts to ca. $0.625 \mathrm{eV}$.
The calculated band width in the fifth neighbor's scheme leads to $82 \%$ of $\Delta \varepsilon_{v}^{\infty}$ while the $j=1$ approximation gives only $32 \%$ of the extrapolated "exact" HF dispersion. One-electron calculations of the WH-type predict a nearly dispersionless $\varepsilon(k)$ curve (band width: ca. $0.05 \mathrm{eV}$ ).

On the basis of the material of Table 1 and guided by the above mentioned WH band width it is clear that the $j$-dependent broadening of the occupied dispersion curves is exclusively caused by the exchange part of the mean-field operator. In accordance with the theoretical analysis in section 3 the width of the lowest unfilled band $(c)$ is not strongly influenced by the extension of the lattice summation. On the other side, it can be seen that changes in the $j$-array are accompanied by shifts of the absolute positions of the HF bands that are roughly comparable in the filled and virtual Fermiseas. The center of gravity of the filled bands of $\mathbf{2}$ is shifted by ca. $0.45 \mathrm{eV}$ to higher energies $(3: 0.35 \mathrm{eV})$, the conduction band of Ni(TMP) is destabilized by $0.25 \mathrm{eV}(3: 0.16 \mathrm{eV})$.

The exchange-influence on the $\Delta \varepsilon$ figures of $\mathbf{2}$ is much smaller. The width of the two highest filled bands is enlarged by only ca. $10 \%$ if the lattice summation is extended from the nearest neighbor's approximation to $j=5$. The absolute magnification 
of $\Delta \varepsilon_{i}$ of $\mathbf{2}$ due to LR exchange contributions is less than $0.1 \mathrm{eV}$ while a $0.3 \mathrm{eV}$ correction $(j=1 \rightarrow j=5)$ is predicted in 3. An explanation of the weaker $j$ dependence of $\Delta \varepsilon_{r}$ of $\mathbf{2}$ as compared to $\mathbf{3}$ is given below.

The band gap $\Delta E_{\mathrm{G}}$ is also a function of the spatial extension of the exchange summation. The theoretically determined variation of $\Delta E_{\mathrm{G}}\left(=\Delta \Delta E_{\mathrm{G}}\right)$ in 2 as a function of $j$ is smaller than $0.15 \mathrm{eV}$. Larger modifications are encountered in the highly puckered $\mathrm{Ni}$ (II) model 3. The nearest neighbor's scheme leads to a forbidden gap of $4.17 \mathrm{eV}$; this value is reduced to $3.85 \mathrm{eV}$ if five adjacent building blocks are taken into account. The $j$-dependence of $\Delta E_{\mathrm{G}}$ of $\mathbf{3}$ is displayed in Figure 20. A rough extrapolation to $N \rightarrow \infty$ results in an "exact" HF band gap of $3.75 \mathrm{eV}$. The proper treatment of the electronic exchange reduces $\Delta E_{\mathrm{G}}$ by an amout of $0.42 \mathrm{eV}$. Such uncertainties cannot be neglected in semiconducting materials with energy gaps of the order of some tenth of an eV. Reliable approximations for the determination of the exchange potential (i.e. spatial extension of the lattice summation) are therefore an absolute prerequisite in SCF HF crystal orbital approaches to semiconductors with narrow dispersion curves.

The $\varepsilon(k)$ dispersions of the valence bands $(v)$ of $\mathbf{2}$ and 3 are graphically shown in the Figs. 21 and 22. The numerical results of five separate tight-binding calculations per 1D stack are collected in the two displays. The exchange-induced nonlinearities of the HF bands of $\mathbf{3}$ exceed the perturbations in $\mathbf{2}$. But even in the Ni(TMP) model $\mathbf{2}$ significant deviations of the $\varepsilon(k)$ curves (reference: idealized tightbinding relation in the nearest neighbor's scheme) are predicted as soon as the lattice summation is extended beyond the direct neighbors. The Ni(TMP) chain is an example in the class of narrow-band materials showing opposite slopes of the $k$-modulated resonance and exchange energies. These contrary effects lead to the turn of $\nabla_{\varepsilon}(k)$ at larger values of the wave vector $k(j \neq 1$, see Figure 21$)$.

Stronger nonlinearities and deviations from usual tight-binding curves are found for the valence band of 3 (Figure 22). The reduced one-electron part of the CO Hamiltonian allows for an amplification of exchange-dependent phenomena. The various $\varepsilon(k)$ curves in Fig. 22 are not unlike to the dispersions presented in connection with the model calculations of Section 3. The $\varepsilon(k)$ curve associated to the $j=1$

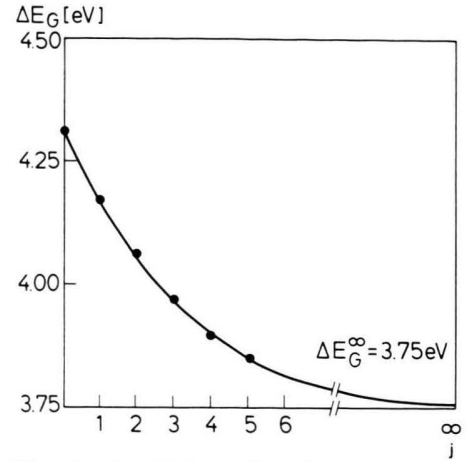

Fig. 20. Forbidden band gap $\Delta E_{\mathrm{G}}$ of $\mathbf{3}$ as a function of the lattice sum dimension employed in the semiempirical SCF HF INDO CO approximation. $\Delta E_{\mathrm{G}}^{\infty}$ is the "exact" HF gap which has been extrapolated to $N \rightarrow \infty$.
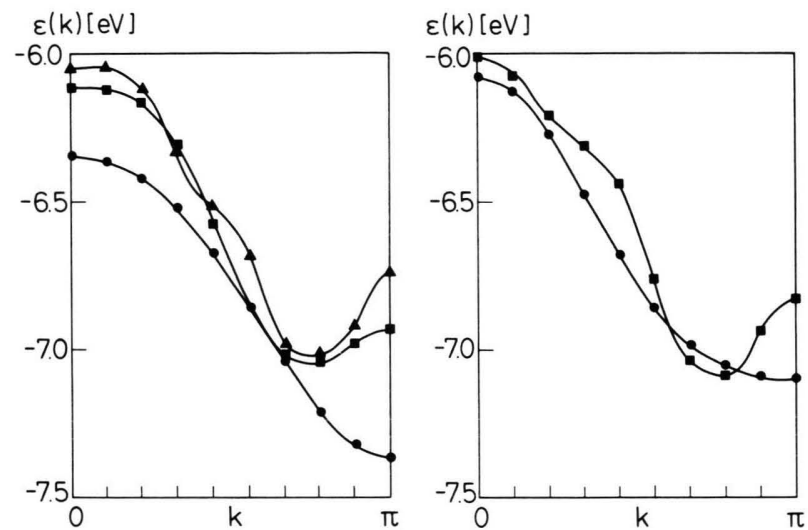

Fig. 21. HF dispersions of the valence band of 2 as a function of the lattice sum dimension $j$. On the lhs. $\varepsilon(k)$ relations derived for ungerade $j$-arrays $(j=1,3$ and 5$)$ are shown while the gerade members $(j=2$ and 4$)$ are collected on the rhs. The idealized tight-binding band with $j=1$ has been labeled by full circles, the $j=3$ curve by squares and the $j=4$ dispersion by triangles. Rhs.: $j=2:$ $j=4$ :
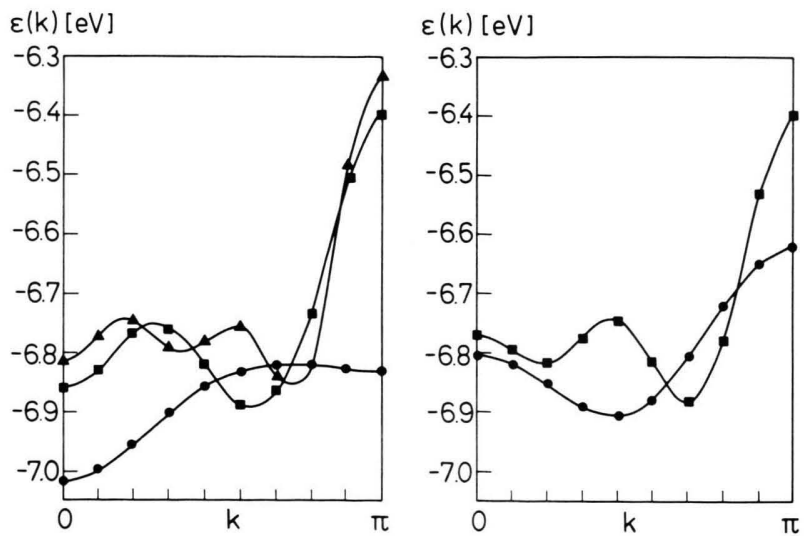

Fig. 22. HF dispersion of the valence band of $\mathbf{3}$ as a function of the lattice sum dimension $j$. See legend Figure 21 

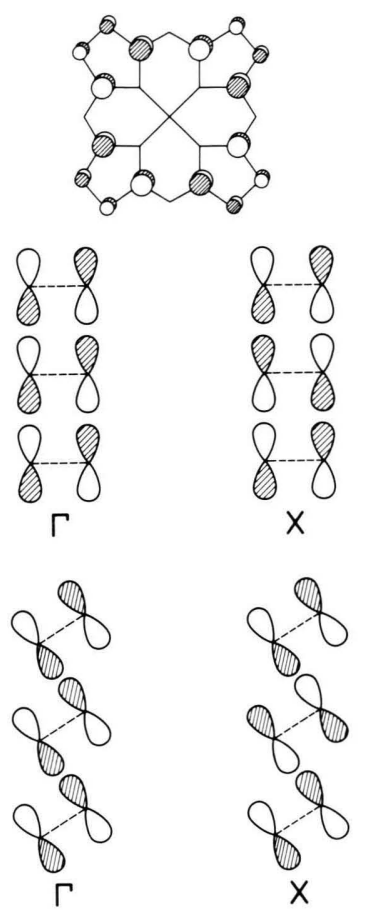

Fig. 23. Schematic representation of the $\mathrm{CO}$ amplitudes of the highest filled band of eclipsed porphyrinato nickel(II), $\mathrm{Ni}(\mathrm{P})$ (top). In the middle we have schematized the interaction between the $\pi$ AO's at $C_{1}$ and $C_{4}$ or $C_{2}$ and $C_{3}$, respectively, into the direction of the stacking axis in the limit of small (vanishing) puckering deformations around the central Ni sites. The $\Gamma$-states are determined by antibonding linear combinations of $\pi$ orbitals localized at neighboring building blocks. The $\mathrm{X}$-states are characterized by in-phase interactions between topologically identical ligand atoms in adjacent stacking units. Bottom: $\pi-\pi$ interaction into the direction of the 1D axis for highly puckered ligand rings. The out-of-plane deformation allows for stabilizing interactions between $C_{1}\left(j\right.$ th cell) and $C_{4}$ (cell: $j \pm 1$ ) etc. at the $\Gamma$-point but leads to antibonding relations at the edge of the Brillouin zone. It is thus clear that the tight-binding (one-electron) interaction in $\mathbf{2}$ and $\mathbf{3}$ is responsible for different energy gradients. The broken lines in the two lower representations symbolize the meanplanes of the pyrrole rings.

approximation indicates the presence of positive interference effects of both $k$-dependent contributions to the HF operator. The gradients associated to the one- and two-electron potentials are positive.

The divergent $\varepsilon(k)$ relations derived for the valence bands of $\mathbf{2}$ and $\mathbf{3}$ can be explained on the basis of Fig. 23 which contains a schematic representation of the $\mathrm{CO}$ amplitudes of the highest filled band in the porphyrinato nickel(II) series. The CO microstates of ligand $\pi$ character transform according to $\mathrm{a}_{1 u}$ symmetry if the irreducible representa- tions of the spatial point group $\mathrm{D}_{4 h}$ are employed ( $\mathrm{a}_{2}$ under $\mathrm{C}_{4}$ symmetry). The phases of the $\pi$ orbitals allow for a stabilization of $2 \cos (k j) t_{\mu_{0} \mu_{\text {, }}}$ with increasing values of the $k$ vector (small or vanishing puckering modes around $\mathrm{Ni}$ assumed). The $\Gamma$-states of $(v)$ are therefore determined by antibonding $\pi$ interactions between topologically identical ligand centers in adjacent stacking units. In-phase relations between the $\pi$ AO's are allowed at the $X$-point. The tight-binding matrix elements create a dispersion pattern with a negative gradient. This is schematized in the middle of Figure 23. Strong puckering deformations within the $\mathrm{Ni}(\mathrm{P})$ units are accompanied by reductions of the internuclear separations between $\mathrm{C}_{1}\left(j\right.$ th cell) and $\mathrm{C}_{4}(j \pm 1)$ or $\mathrm{C}_{2}\left(j^{\prime}\right.$ th cell) and $\mathrm{C}_{3}(j \pm 1)$ etc. that belong always to neighboring molecular building blocks. The symmetry properties of the $a_{1 u}\left(a_{2}\right)$ crystal orbitals within the pyrrole rings (i.e. antisymmetric behaviour with respect to the mirror plane spanned by $N$ and $\mathrm{C}_{2} / \mathrm{C}_{3}$ ) allows for stabilizing interligand interactions at $\Gamma$ while out-of-phase relations are encountered at the edge of the Brillouin zone. The relevant intercell coupling is schematized at the bottom of Figure 23. The opposite gradients of the valence bands of $\mathbf{2}$ and $\mathbf{3}$ are thus a direct consequence of the increasing tetrahedral deformation around $\mathrm{Ni}$ in 3 .

The exchange contributions to the HF operator lead of course to an increase in the width of all filled bands. This is verified in Table 4 for the occupied "Ni $3 \mathrm{~d}$ bands" of the $3 \mathrm{~d}^{8}(\mathrm{Ni} 3 \mathrm{~d} \sigma=$ $3 \mathrm{~d}_{z^{2}}, 3 \mathrm{~d} \pi=3 \mathrm{~d}_{x z} / 3 \mathrm{~d}_{y z}, 3 \mathrm{~d} \delta=3 \mathrm{~d}_{x^{2}-y^{2}}$ ) systems 2 and 3. We have summarized the net energy intervals $\tilde{\Delta \varepsilon}$ where microstates with predominant $3 \mathrm{~d} \sigma$, $3 \mathrm{~d} \pi$ and $3 \mathrm{~d} \delta$ amplitudes are found. The $\tilde{\Delta \varepsilon}$ elements are therefore not necessarily identical with the width, $\Delta \varepsilon$, of a single $\mathrm{HF}$ dispersion as $k$ dependent hybridization with ligand bands allows for distributions of the relevant "Ni $3 \mathrm{~d}$ states" over several energy bands (see discussion in [29]). For the $3 \mathrm{~d} \sigma$ states two $\Delta \varepsilon$ intervals have been given in Table 4. The splitting into two energetically separated $\mathrm{Ni} 3 \mathrm{~d}$ blocks results from strong covalent interactions between the $3 \mathrm{~d}_{z^{2}}$ AO's and lone-pair orbitals of the $N$ ligands. This coupling leads to one $\varepsilon(k)$ region characterized by bonding (B) in-phase interactions between the central site and the nitrogen atoms as well as to an antibonding (AB) out-ofphase counterpart [48, 49]. 
Table 4. Band structure properties of the four occupied "Ni $3 \mathrm{~d}$ bands" $3 \mathrm{~d} \sigma\left(=3 \mathrm{~d}_{z^{2}}\right), 3 \mathrm{~d} \pi\left(=3 \mathrm{~d}_{x z} / 3 \mathrm{~d}_{y z}\right)$ and $3 \mathrm{~d} \delta$ $\left(=3 \mathrm{~d}_{x^{2}-y^{2}}\right)$ of $\mathbf{2}$ and $\mathbf{3}$ as a function of the employed $j$-array. Only the results for $j=1,3$ and 5 are given in the table. We have summarized the band energies at the marginal $k$-points $\Gamma$ and $\mathrm{X}$. The $\Delta \varepsilon$ labels correspond to those energy intervals where microstates with large $\mathrm{Ni} 3 \mathrm{~d}$ amplitudes are found. The $\Delta \varepsilon$ elements are not always identical with the width of a single $\varepsilon(k)$ curve, $\Delta \varepsilon$, as the $3 \mathrm{~d}$ microstates are sometimes smeared over several HF bands (i.e. hybridization with ligand bands). In the case of the $3 \mathrm{~d} \sigma$ states two $\Delta \varepsilon$ intervals have been given in the table; the first one corresponds to an antibonding (AB) linear combination with ligand orbitals, the second one to the in-phase, bonding (B) counterpart (see text). All values in $\mathrm{eV}$.

\begin{tabular}{|c|c|c|c|c|c|}
\hline \multicolumn{3}{|l|}{ Model } & $\begin{array}{l}j=1 \\
-12.08 \\
-11.39\end{array}$ & $\begin{array}{l}j=3 \\
-11.81 \\
-10.86\end{array}$ & $\begin{array}{l}j=5 \\
-11.73 \\
-10.66\end{array}$ \\
\hline $23 \mathrm{~d} \sigma=3 \mathrm{~d}_{z^{2}}$ & $\mathrm{AB}$ & $\begin{array}{l}\varepsilon(0) \\
\varepsilon(\pi) \\
\widetilde{\Delta \varepsilon} \\
\varepsilon(0) \\
\varepsilon(\pi) \\
\widetilde{\Delta \varepsilon}\end{array}$ & $\begin{array}{r}-12.08 \\
-11.39 \\
0.69 \\
-14.87 \\
-14.15 \\
0.72\end{array}$ & $\begin{array}{r}-11.81 \\
-10.86 \\
0.95 \\
-14.58 \\
-13.67 \\
0.91\end{array}$ & $\begin{array}{r}-11.73 \\
-10.66 \\
1.07 \\
-14.51 \\
-13.47 \\
1.04\end{array}$ \\
\hline $3 \mathrm{~d} \pi=3 \mathrm{~d}_{x z} / 3 \mathrm{~d}_{y z}$ & & $\begin{array}{l}\varepsilon(0) \\
\varepsilon(\pi) \\
\widetilde{\Delta \varepsilon}\end{array}$ & $\begin{array}{r}-13.63 \\
-12.89 \\
0.74\end{array}$ & $\begin{array}{r}-13.29 \\
-12.35 \\
0.94\end{array}$ & $\begin{array}{r}-13.21 \\
-12.14 \\
1.07\end{array}$ \\
\hline $3 \mathrm{~d} \delta=3 \mathrm{~d}_{x^{2}-y^{2}}$ & & $\begin{array}{l}\varepsilon(0) \\
\varepsilon(\pi) \\
\widehat{\Delta \varepsilon}\end{array}$ & $\begin{array}{r}-13.36 \\
-12.81 \\
0.55\end{array}$ & $\begin{array}{r}-13.10 \\
-12.25 \\
0.85\end{array}$ & $\begin{array}{r}-13.02 \\
-12.04 \\
0.98\end{array}$ \\
\hline \multirow[t]{2}{*}{$33 \mathrm{~d} \sigma=3 \mathrm{~d}_{z^{2}}$} & $\mathrm{AB}$ & $\begin{array}{l}\varepsilon(0) \\
\varepsilon(\pi) \\
\widetilde{\Delta \varepsilon}\end{array}$ & $\begin{array}{r}-12.28 \\
-11.65 \\
0.63\end{array}$ & $\begin{array}{r}-12.10 \\
-11.27 \\
0.83\end{array}$ & $\begin{array}{r}-12.05 \\
-11.11 \\
0.94\end{array}$ \\
\hline & B & $\begin{array}{l}\varepsilon(0) \\
\varepsilon(\pi) \\
\widetilde{\Delta \varepsilon}\end{array}$ & $\begin{array}{r}-14.99 \\
-14.61 \\
0.38\end{array}$ & $\begin{array}{r}-14.82 \\
-14.03 \\
0.79\end{array}$ & $\begin{array}{r}-14.77 \\
-13.86 \\
0.91\end{array}$ \\
\hline $3 \mathrm{~d} \pi=3 \mathrm{~d}_{x z} / 3 \mathrm{~d}_{y z}$ & & $\begin{array}{l}\varepsilon(0) \\
\varepsilon(\pi) \\
\widetilde{d \varepsilon}\end{array}$ & $\begin{array}{r}-13.99 \\
-13.14 \\
0.85\end{array}$ & $\begin{array}{r}-13.86 \\
-12.81 \\
1.05\end{array}$ & $\begin{array}{r}-13.76 \\
-12.60 \\
1.16\end{array}$ \\
\hline $3 \mathrm{~d}=3 \mathrm{~d}_{x^{2}-y^{2}}$ & & $\begin{array}{l}\varepsilon(0) \\
\varepsilon(\pi) \\
\widetilde{\Delta \varepsilon}\end{array}$ & $\begin{array}{r}-13.61 \\
-13.09 \\
0.52\end{array}$ & $\begin{array}{r}-13.42 \\
-12.71 \\
0.71\end{array}$ & $\begin{array}{r}-13.38 \\
-12.55 \\
0.83\end{array}$ \\
\hline
\end{tabular}

The numerical results in Table 4 are in line with the foregoing discussion, i.e. the Ni $3 \mathrm{~d}$ distributions in $\mathbf{2}$ and $\mathbf{3}$ are broadened under the influence of the electronic exchange. The width of the variation, however, is smaller than the $\Delta \varepsilon$ intervals given in Table 3. This is expected as a result of the various forbidden crossing regions of the $\varepsilon(k)$ curves (i.e. metal-ligand hybridization) that lead to an intrinsic ( $j$-independent) magnification of the distribution of certain CO microstates.

To sum up, the numerical $\mathrm{CO}$ data presented in the Sects. 3 and 5, respectively, allow for the formulation of general conclusions that are neither restricted to specific classes of narrow-band materials nor subject to the theoretical details of the employed SCF HF CO variant:
1) The HF energy bands (of narrow-band materials) are broadened via the $k$-dependent exchange contribution to the HF operator. This two-electron effect is restricted to the filled bands of the polymer or solid and goes beyond the nearest neighbors. The exchange-dependent increase of HF band widths exceed the dispersions via the kinetic hopping terms up to one order of magnitude in the limit of small (or vanishing) covalent interactions between adjacent stacking units.

2) The broadening of the band width is accompanied by strong perturbations of the structure of the $\varepsilon(k)$ curves (reference tight-binding band). Irrespective of the adopted $j$-array enlarged gradients are encountered in the neighborhood of the $X$-point $(\Gamma$-point) while flattened $\varepsilon(k)$ intervals are predicted in the vicinity of the zone-center (zone-edge). The former band shape is valid for $D_{\mu \nu}^{ \pm}(j)$ matrices that decay monotonously while oscillating (alternant) intercell bond-order matrices create the dispersion pattern given in parentheses.

3) The restriction to finite neighbor's approximations, which are part of all numerical CO investigations of "real solids", is responsible for nonlinearities in the HF bands. This makes one-electron properties at a certain $k$-point strongly dependent on the dimension of the lattice summation. Effective mass-parameters or group velocities calculated at a given $k$-point thus may be wrong even for qualitative purposes. Such numerical errors are restricted to $k$-dependent properties associated to microstates of the solid. Sufficiently extended lattice summations render possible accurate evaluations of total energies (within a preselected computational framework) and fair results of averaged one-electron properties (integration over the $k$-figures in the Brillouin zone; e.g., band widths, absolute positions of $\mathrm{HF}$ dispersions). A necessary prerequisite to guarantee a numerically reliable access to these theoretical figures is however an integration procedure for the $\mathrm{D}_{\mu \nu}^{ \pm}(j)$ which prevents increasing relative errors with enlarged separations between the interacting cells.

4) The "oscillations" (higher Fourier components) in some of the $\varepsilon(k)$ diagrams in the sections 3 and 5 must be traced back to the truncations in the expansion of the exchange potential; they vanish in the limit $N \rightarrow \infty$ and are smoothed by electronic correlations (see below). 
5) The analytic structures of HF dispersions of narrow-band materials are intermediate between the $\varepsilon(k)$ relations of idealized tight-binding bands (determined by the dominating one-electron part of the mean-field operator with its exponential decay), on one side, and metallic systems, on the other. Divergent $\varepsilon(k)$ gradients at the Brillouin zone characterize the "exact" HF solutions of metals. Numerical sources preventing the detection of this behaviour have been discussed by Monkhorst [13].

6) The occurrence of the exchange-dependent perturbations of $\mathrm{HF}$ bands is restricted to solids or polymers with narrow dispersions (ca. $\leqq 1 \mathrm{eV})$. Enlarged band widths via covalent resonance integrals allow for a (perfect) masking of the exchange influence.

\section{Retardation of Exchange Contributions via Electronic Correlations and Electron-Phonon Coupling}

On the basis of uncorrected mean-field values $\Delta \varepsilon_{v}$ for $\mathbf{1}\left(\Delta \varepsilon_{v}=2.340 \mathrm{eV}\right.$, see Ref. [26]), $2\left(\Delta \varepsilon_{v}=1.080 \mathrm{eV}\right)$ and $3\left(\Delta \varepsilon_{v}=0.625 \mathrm{eV}\right)$ operational parameters $g$ (correlation strength) are derived that are in strong contradiction to the experimental deductions of the Refs. [34-37]:

$$
g=U / 4 t=U / \Delta \varepsilon_{v} .
$$

The relevant on-site repulsions $U$ in the porphyrinato series are $1.2 \mathrm{eV}$ for the tetrabenz-derivatives $\mathbf{1}$ and $\mathbf{3}$ and $2.7 \mathrm{eV}$ for the small-ring polymer $\mathbf{2}$. The following $g$ numbers are therefore calculated for $\mathrm{Ni}(\mathrm{TBP}), \mathrm{Ni}(\mathrm{TMP})$ and $\mathrm{Ni}(\mathrm{OMTBP})$, respectively:

$$
\begin{array}{ll}
\mathrm{Ni}(\text { (BВP) }: & g=1.2 / 2.340 \cong 0.5 ; \\
\mathrm{Ni}(\mathrm{TMP}): & g=2.7 / 1.080 \cong 2.5 ; \\
\mathrm{Ni}(\text { OMTBP }): & g=1.2 / 0.625 \cong 1.9 .
\end{array}
$$

The corrrelation strength in $\mathbf{3}$ (derived in the framework of the HF approximation) is far from the experimentally verified atomic limit $g \rightarrow \infty$ and is even smaller than the $g$ factor of $\mathbf{2}$.

In order to calculate reliable $\Delta \varepsilon_{v}$ (or $t$ ) elements in narrow-band materials it is absolutely necessary to consider the influence of electronic correlations and electron-phonon coupling on the width of the bare HF dispersions; both contributions tend to reduce the one-particle band width $\Delta \varepsilon$. The magnitude and the importance of quasi-particle (QP) corrections beyond the HF description (i.e. electronic correlations) has been investigated by several authors [65-73]. Numerical estimates of the many-body corrections on the positions and widths of HF dispersions require two incisive approximations. One important a priori assumption is the fragmentation of the total QP corrections into LR and SR contributions. The former QP energies are associated with a basis of spatially uncorrelated Bloch orbitals $\psi_{l}(k)$. The SR reorganizations (electronic relaxations and correlations, see [72] and [73]), on the other side, have to be calculated for the molecular building blocks of the low-dimensional material (Wannier-type orbitals). Both QP elements are conveniently derived in the form of virtual two-band (two-orbital) excitons (configuration interaction (CI) picture in the language of molecular quantum mechanics).

Under the assumption of the validity of these approximations (unique subdivision into LR and SR corrections, description of electronic correlations by two-band (two-orbital) excitons), it is possible to define QP levels for occupied and virtual bands via $\mathrm{HF}$ one-electron energies as well as self-energy corrections that are either defined in the delocalized Bloch basis or by spatially localized orbital wave functions of the Wannier-type:

$$
\begin{aligned}
\varepsilon_{\mathrm{occ}}^{\mathrm{QP}}(k)= & \varepsilon_{\mathrm{occ}}(k)+\sum_{\mathrm{H}, \mathrm{occ}}^{(M-1)}+\sum_{\mathrm{E}, \mathrm{occ}}^{(M)} \\
& +M_{\mathrm{H}, \mathrm{occ}}^{\left(M^{\prime}-1\right)}+M_{\mathrm{E}, \mathrm{occ}}^{\left(M^{\prime}\right)}, \\
\varepsilon_{\mathrm{vir}}^{\mathrm{QP}}(k)= & \varepsilon_{\mathrm{vir}}(k)+\sum_{\mathrm{H}, \mathrm{vir}}^{(M)}+\sum_{\mathrm{E}, \mathrm{vir}}^{(M+1)} \\
& +M_{\mathrm{H}, \mathrm{vir}}^{\left(M^{\prime}\right)}+M_{\mathrm{E}, \mathrm{vir}}^{\left(M^{\prime}+1\right)},
\end{aligned}
$$

$M\left(M^{\prime}\right)$ : Number of electrons in the uncharged polymer (neutral stacking unit).

$\Sigma_{\mathrm{H}, \text { occ }}^{(M-1)}$ is the self-energy of the occ'th delocalized Bloch-hole and is of crucial importance for the reduction of the band width via QP interactions. $\sum_{\mathrm{E}, \text { occ }}^{(M)}$ is the corresponding electron self-energy. $\sum_{\mathrm{H}, \mathrm{vir}}^{(M)}$ and $\sum_{\mathrm{E}, \mathrm{vir}}^{(M+1)}$ are the counterparts of $\Sigma_{\mathrm{H}, \text { occ }}^{(M-1)}$ and $\sum_{\mathrm{E}, \text { occ }}^{(M)}$, respectively, in the virtual subspace. The SR corrections are abbreviated by $M_{\mathrm{H}, \text { occ }}^{\left(M^{\prime}-1\right)}$, $M_{\mathrm{E}, \text { occ }}^{\left(M^{\prime}\right)}, M_{\mathrm{H}, \mathrm{vir}}^{\left(M^{\prime}\right)}$ and $M_{\mathrm{E}, \mathrm{vir}}^{\left(M^{\prime}+1\right)}$. $M_{\mathrm{E}, \text { occ }}^{\left(M^{\prime}\right)}$ describes the loss of ground state (pair-)correlation due to the removal of one electron. $M_{\mathrm{H}, \text { occ }}^{\left(M^{\prime}-1\right)}$ contains SR reorganizations that can be decomposed into orbital relaxation as well as the many-body response to these mean-field processes (see [72], [74] and [75]). Analogous physical quantities are described by the 
two remaining SR self-energy corrections which act on the unfilled one-particle space (particle-hole symmetry). A detailed discussion of the physical significance of the various self-energy parameters in (50) and (51) can be found in the [72] and [73].

$$
\begin{aligned}
& \sum_{\mathrm{H} . \text { occ }}^{(M-1)} \text { is given by } \\
& \sum_{\mathrm{H}, \mathrm{occ}}^{M-1)}=-\sum_{\substack{J \\
J, L \neq \mathrm{occ}}} \sum_{\substack{A \\
\sum_{J}}} \frac{\left\langle\psi_{J} \psi_{L}\left(1-P_{12}\right) r_{12}^{-1} \psi_{\mathrm{occ}} \psi_{A}\right\rangle^{2}}{\varepsilon_{J}+\varepsilon_{L}-\varepsilon_{\mathrm{occ}}-\varepsilon_{A}} .
\end{aligned}
$$

The capital letters $J, L$ and $A$ are compound indices containing both band $(j, l, a)$ and $k\left(k_{j}, k_{l}, k_{a}\right)$ coordinates; $J$ and $L$ span the filled HF states and $A$ the virtual bands. The $\psi_{J}$, etc. are Bloch orbitals expressed by the more compact notation $\left(\psi_{j}\left(k_{j}\right)\right)$. $\left(1-P_{12}\right)$ is the convenient permutation operator; the matrix elements in the numerator of (52) are thus of the Coulomb-exchange type. The virtual excitations leading to the reduction of $\Delta \varepsilon_{r}$ in the QP description are symbolized in the schematic diagram of Figure 24. Microstates at the bottom of the (valence) band are stronger shifted to higher energies than levels at the top of the band. It is seen that the virtual scattering processes from the top to the bottom of a band allow for a partial reduction of those energy differences in (52) that are at least identical with the forbidden gap. It is obvious-that

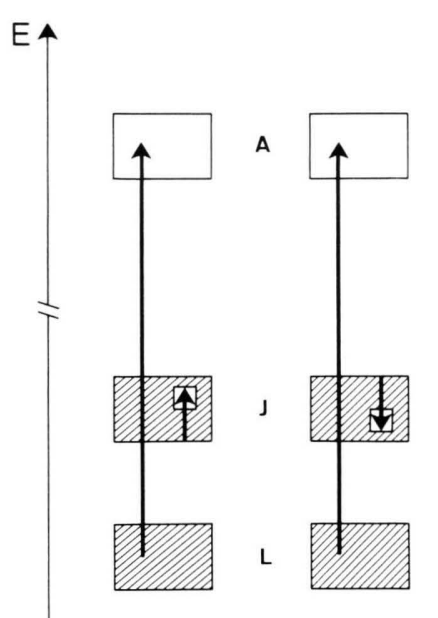

Fig. 24. Schematic representation of the virtual scattering processes leading to the reduction of the band width, $\Delta \varepsilon$, in the filled Fermi-sea. The opposite signs of the two singleparticle excitations in the diagram on the rhs. of the figure reduce the energy denominators of (52), i.e. the states at the bottom of the band are stronger shifted to higher $\varepsilon(k)$ values. Occupied states have been labeled by hatched areas while empty one-particle levels are symbolized by blank bars. the exchange-induced "oscillations" in $\varepsilon(k)$ curves (see Sects. 3 and 5) are partially suppressed by the virtual two-band excitons displayed in Figure 24.

If it is assumed that the four-index integrals in (52) are $k$-independent for the microstates of the valence band(s) (absence of hybridization effects), it is possible to estimate the percentage reduction of $\Delta \varepsilon_{r}, f_{\mathrm{red}, t}$, by means of the equation

$f_{\text {red }, r} \approx \frac{2 \varepsilon_{r}(\text { top })-\varepsilon_{r}(\text { bottom })-\varepsilon_{c}(\text { bottom })}{2 \varepsilon_{r}(\text { top })-\varepsilon_{r}(\text { top })-\varepsilon_{c}(\text { bottom })}$,

$\Delta \varepsilon_{r}(\mathrm{el}-\mathrm{pol})=\Delta \varepsilon_{r} \cdot f_{\text {red, } r}$.

$\varepsilon_{r}$ (top) is the $\varepsilon_{r}(k)$ value at the top of the $v^{\prime}$ th dispersion curve and $\varepsilon_{v(c)}$ (bottom) symbolizes the one-particle energy at the bottom of the valence or conduction band. The "new" band width emerging from the formation of an electronic polaron is given in (54). The approximate relation of (53), however, is only valid if the inequality

$$
\Delta E_{\mathrm{G}}>\Delta \varepsilon_{r}
$$

if fulfilled.

Equation (55) guarantees the convergence of the $\Sigma_{\mathrm{H}, \mathrm{occ}}^{(M-1)}$ expansion. The results summarized in Table 3 show that this relation is fulfilled for both $1 \mathrm{D}$ chains 2 and 3. Nevertheless, we will restrict the subsequent analysis to the "pathological" model polymer 3. Insertion of the relevant $\varepsilon(k)$ figures from Table 3 into (53) and (54) leads to a reduction in the width of the band that amounts to ca. 17\% $\left(f_{\mathrm{red}, t}=0.83\right), \Delta \varepsilon_{t}$ (el-pol) is $0.52 \mathrm{eV}$.

In the theoretical formalism leading to $\Delta \varepsilon_{v}$ (el-pol) we have assumed that the one-particle energies of the canonical HF bands are sufficient approximations to determine the various self-energy corrections (e.g. $\sum_{\mathrm{H}, \text { occ }}^{M-1)}$ of (52)). This procedure neglects however all shifts of the QP energies due to local reorganization processes dressing the charge carriers (SR domain) as well as LR corrections that would enter the rhs. of (52) in a self-consistent variant of the perturbational expansion. An improved definition of $\sum_{\mathrm{H}, \text { occ }}^{(M-1)}$ is therefore given by

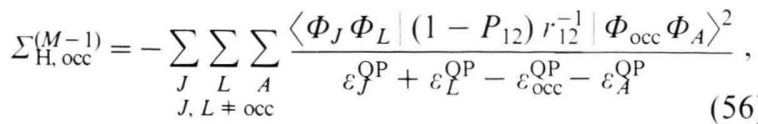

where the bare HF eigenvalues are replaced by "exact" QP energies; the Bloch orbitals $\psi_{J}$ concordantly have been detached by quasi-particle wave functions $\Phi_{J}$. 

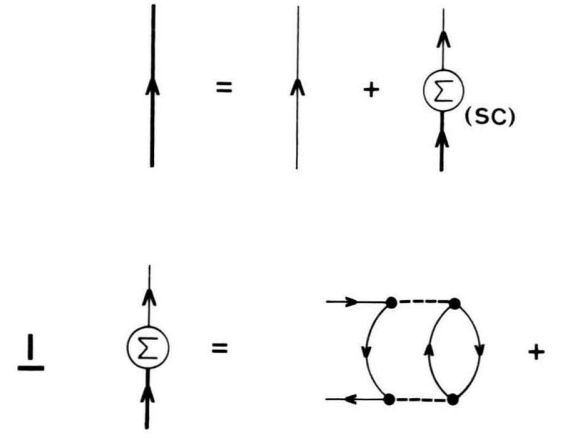

II
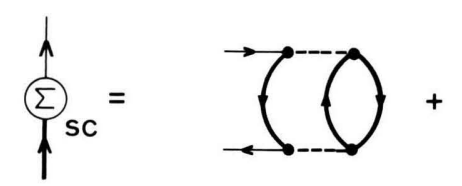

Fig. 25. Diagrammatic representation of the Dyson equation (top). I: Definition of the self-energy part by means of bare mean-field energies. II: Self-consistent determination of the self-energy corrections. The HF lines have been replaced by QP lines that contain both long-range and short-range reorganizations.

The solutions of (56) are feasible by iteration where improved QP energies are defined by the aforementioned self-energy elements $\Sigma_{\mathrm{H}, \text { occ }}^{(M-1)}$, etc. The fundamental difference between the relations (52) and (56) is shown in Fig. 25 by well-known diagrammatic representations. In the figure it is supposed that the QP corrections on the rhs. of (50) and (51) are solutions of Dyson-type equations [76]. The two lower diagrams in Fig. 25 differ in so far as the HF lines of I (associated to (52)) are replaced by QP lines (II, (56)).

The straightforward extensions of (53) and (54) are given by

$f_{\text {red }, v}^{\prime}=\frac{2 \varepsilon_{v}^{\mathrm{QP}}(\text { top })-\varepsilon_{v}^{\mathrm{QP}}(\text { bottom })-\varepsilon_{c}^{\mathrm{QP}}(\text { bottom })}{2 \varepsilon_{v}^{\mathrm{QP}}(\text { top })-\varepsilon_{v}^{\mathrm{QP}}(\text { top })-\varepsilon_{c}^{\mathrm{QP}}(\text { bottom })}$,

$\Delta \varepsilon_{v}^{\mathrm{QP}}=\Delta \varepsilon_{v} f_{\mathrm{red}, v}^{\prime} ;$

these relations allow for the evaluation of QP corrections under the inclusion of LR and SR reorganizations.

The large unit cell dimension of $\mathbf{3}$ prevents first principle determinations of $\Sigma_{\mathrm{H} \text {, occ }}^{(M-1)}$, etc. In order to allow for a comparison between (52) and (53), on one side, as well as (57) and (58), on the other, we make use of experimental excitation energies (energy denominators) available for macrocyclic $\mathrm{Ni}$ (II) materials [77, 78]. We adopt an experimental gap of $1.5 \mathrm{eV}$ and retain the $\Delta \varepsilon_{v}$ parameter of $0.625 \mathrm{eV}$. This leads to an effective QP band width $\Delta \varepsilon_{v}^{\mathrm{QP}}$ of $0.36 \mathrm{eV}$; the LR and SR reorganizations reduce the HF dispersion by ca. $42 \%$. Nearly one half of the exchangeinduced broadening of the band is thus compensated by electronic correlations. The $\Delta \varepsilon_{v}$ (el-pol) and $\Delta \varepsilon_{l}^{\mathrm{QP}}$ figures are nevertheless significantly larger than the dispersion derived by one-electron calculations of the WH-type $(0.05 \mathrm{eV})$. The comparison between $\Delta \varepsilon_{v}$ (el-pol) and $\Delta \varepsilon_{v}^{\mathrm{QP}}$ indicates that both types of many-body interactions (i.e. spatially correlated reorganizations dressing local holes and coherent scattering processes leading to LR polarizations of the medium) have an important control on the widths of dispersion curves.

The weak intercell coupling in $\mathbf{3}$ (and 2) leads to localization times

$$
\tau=\hbar / \Delta \varepsilon=\hbar / 4 t
$$

of the charge carriers that are comparable with the frequencies of intramolecular vibrations. Therefore significant interactions between electrons and optical phonon modes are expected [79]. The subsequent analysis concordantly is restricted to the (linear) interaction between (localized) carriers and intramolecular optical phonon modes. We start with the (molecular) model Hamiltonian defined by

$$
\begin{aligned}
& H=H_{\mathrm{el}}+H_{\mathrm{ph}}+H_{\mathrm{el}-\mathrm{ph}}, \\
& H_{\mathrm{el}}=F+H_{\mathrm{res}}, \\
& F=\sum_{i} \varepsilon_{i} a_{i}^{+} a_{i}
\end{aligned}
$$

and make use of the formalism of second quantization. $H_{\mathrm{el}}$ symbolizes the electronic Hamiltonian which has been divided into the mean-field part $F$ and the residual interaction $H_{\text {res }}$ (QP corrections). The $a_{i}^{+}\left(a_{i}\right)$ are creation (destruction) operators of the $i$ 'th one-electron wave function. The pure phononic part is given by

$$
H_{\mathrm{ph}}=\sum_{I} \hbar \omega_{I} b_{I}^{+} b_{I},
$$

where $\omega_{I}$ stands for the energy of the $I$ th normal mode. The $b_{I}^{+}\left(b_{I}\right)$ are creation (annihilation) phonon operators. The linear electron-phonon inter- 
action reads

$$
\begin{aligned}
H_{\mathrm{el}-\mathrm{ph}} & =\sum_{I} G_{I}\left(b_{I}^{+}+b_{I}\right), \\
G_{I} & =\sum_{i} g_{i I} a_{i}^{+} a_{i} .
\end{aligned}
$$

Equation (65) is based on the simplified assumption that modifications of the canonical one-electron wave functions along the $\Gamma$ th totally symmetric normal mode can be neglected (i.e. small variations of the expansion coefficients as a function of geometrical deformations). This approximation should be allowed in connection with the following semiquantitative analysis.

In order to decouple electrons and phonons, we define displacement operators:

$$
\tilde{b}_{I}^{+}=b_{I}^{+}+G_{I} / \hbar \omega_{I} .
$$

By means of the displacement operators $\tilde{b}_{I}^{+}$it is possible to combine $H_{\mathrm{ph}}$ and $H_{\mathrm{el}-\mathrm{ph}}$, respectively, into the form

$$
\begin{aligned}
H_{\mathrm{ph}}+H_{\mathrm{el}-\mathrm{ph}} & =\sum_{I}\left(\hbar \omega_{I} \tilde{b}_{I}^{+} \tilde{b}_{I}-G_{I} G_{I}^{+} / \hbar \omega_{I}\right) \\
& =\sum_{I} \hbar \omega_{I} \tilde{b}_{I}^{+} \tilde{b}_{I}+H_{i I} .
\end{aligned}
$$

$H_{i I}$ symbolizes the renormalization of the electronic Hamiltonian $H_{\text {el }}$ (or $F$ ) via electron-phonon interaction and is given by means of

$$
\begin{aligned}
H_{i I} & =-\sum_{I} \sum_{i}\left[g_{i I}^{2} /\left(\hbar \omega_{I}\right)\right] n_{i}, \\
n_{i} & =a_{i}^{+} a_{i}, \quad n_{i}^{2}=n_{i} .
\end{aligned}
$$

In the (65) and (68) it has been assumed that the one-electron wave functions are defined in a diagonal (canonical) basis. By means of (68) it is possible to incorporate the electron-phonon coupling into renormalized one-electron energies:

$$
\begin{aligned}
\tilde{H} & =H_{i I}+F\left(+H_{\text {res }}\right) \\
& =\sum_{i} \varepsilon_{i} a_{i}^{+} a_{i}-\sum_{I} \sum_{i} g_{i I}^{2} /\left(\hbar \omega_{I}\right) n_{i} \\
& =\sum_{i} \varepsilon_{i} n_{i}-\sum_{I} \sum_{i} g_{i I}^{2} /\left(\hbar \omega_{I}\right) n_{i} .
\end{aligned}
$$

The renormalized one-particle energies $\tilde{\varepsilon_{i}}$ are then defined by

$$
\tilde{\varepsilon_{i}}=\varepsilon_{i}-\sum_{I} g_{i I}^{2} / \hbar \omega_{I} .
$$

This relation shows immediately that the shifted electron-phonon contribution causes a reduction of the band width by forming a Franck-Condon-like vibrational overlap with $\varepsilon_{i}(k)$ or $\varepsilon_{i}^{\mathrm{QP}}(k)$, respectively. If $\tilde{H}$ in (70) would be defined by $F+H_{\text {res }}$, the $\varepsilon_{i}$ figures in (71) have to be replaced by the $\varepsilon_{i}^{\mathrm{QP}}$ elements.

It is much too elaborate to calculate the $g_{i I}^{2} / \hbar \omega_{I}$ parameters for the 62 totally symmetric (A) modes of the Ni(OMTBP) molecule that are allowed to couple linearly to any one-electron state of the $\mathrm{Ni}$ (II) complex. Therefore we make use of theoretically determined electron-phonon interaction parameters of simpler planar $\pi$ systems $[79,80]$. This seems to be an allowed approximation as the important $g_{i I}$ elements are determined by bondstretching vibrations that are highly transferable between topologically related molecules (here: planar $\pi$ systems). Furthermore we assume that the individual $g_{i I}^{2} / \hbar \omega_{I}(i=v)$ corrections can be expressed by an averaged constant $g_{v, a v}^{2} / \hbar \omega_{a v}$ and adopt the mean renormalization derived for the $\mathrm{A}$ modes of TCNQ $(0.003 \mathrm{eV})$ [80]. This approximation for the electron-phonon interaction causes a band narrowing which amounts to $0.186 \mathrm{eV}$. The corrected width of the valence band of $\mathbf{3}$ is therefore $0.17 \mathrm{eV}\left(\Delta \varepsilon_{v}^{\mathrm{QP}}-0.186 \mathrm{eV}\right)$. The "effective" hopping integral is $0.0435 \mathrm{eV}$.

By means of this one-electron parameter and the aforementioned on-site repulsion $U(=1.2 \mathrm{eV})$ it is possible to determine the antiferromagnetic exchange term $J$ (given in (4)) of the distorted porphyrinato $\mathrm{Ni}$ (II) stack 3 . The theoretical $J$ integral of 3 amounts to $13 \mathrm{~cm}^{-1}$, a value that it is in fair agreement with the experimental antiferromagnetic exchange element $J<3 \mathrm{~cm}^{-1}$ and $J<10 \mathrm{~cm}^{-1}$ for the two oxidized modifications of $\mathrm{Ni}$ (OMTBP) with 0.36 or 0.97 electrons removed per stacking unit. There are two additional sources responsible for the small deviation between the theoretical and experimental $J$ integrals: a) the various approximations employed in the semiempirical $\mathrm{CO}$ procedure as well as the necessary computational restriction to $\mathbf{4}$ as model system for the large-ring derivative $\mathbf{3}$; b) the calculation of the exchange contributions for the non-paramagnetic (unoxidized) Ni(OMTBP) chain; the experimental data correspond to a solid that is partly oxidized. In the introduction it had been mentioned that the decay of the electronic exchange is a function of the band occupation scheme.

The experimental effective band width of $\mathbf{3}$ lies in an interval between $0.15 \mathrm{eV}$ and $0.08 \mathrm{eV}$. The cor- 
relation strength in $\mathrm{Ni}(\mathrm{OMTBP})$ derived under the inclusion of QP corrections and electron optical phonon interaction is raised to ca. 7 and differs now significantly from the $g$ elements of $\mathbf{1}$ and $\mathbf{2}$, respectively. The improved $g$ figures of the two latter materials (QP and phononic corrections) are 0.8 and 3.9 .

\section{Conclusions}

It has been the aim of the present contribution to study the influence of the nonlocal Hartree-Fock exchange on the one-electron properties of narrowband materials. We were able to show that weak covalent coupling conditions between the (molecular) building blocks of a polymer or solid allow for a turn of the relative importance of classical tightbinding interactions (i.e. kinetic energy of the electrons) and the Fermi-correlation (antisymmetry of the HF wave function). The hopping integrals decay exponentially, i.e. matrix elements beyond the nearest neighbors are usually of minor significance. The kinetic energy contribution to the net HF dispersion is therefore determined by a single Fourier component $\left(2 \cos (k) t_{\mu_{0} \mu_{1}}\right)$. Nonvanishing interactions beyond the direct neighbors, on the other side, are encountered for the electronic exchange. This imbalance of the one- and two-electron potentials in the $1 \mathrm{D}$ lattice vector $j$ is not restricted to systems with half-filled bands but is also valid in insulators and semiconductors and is responsible for several unexpected features in the one-electron properties of weakly coupled infinite Fermion systems. Only the latter class of solid-state materials (finite band gaps) has been analyzed in the present work. On the basis of the theoretical material presented in $[5,10,11,13]$ and $[14]$, however, it must be expected that the one-particle response to the electronic exchange is reinforced in partly oxidized organometallic chains as a result of the increasing long-range tails of the charge-density bond-order matrices.

Sufficiently extended lattice sums are a prerequisite of reliable SCF HF band structure calculations of systems with small $\Delta \varepsilon$ figures. The influence of the higher order Fourier components to the HF exchange is of crucial importance for calculated one-electron properties in the limit of small or vanishing covalent interactions between adjacent unit cells. A predominance of the $k$-dependent two- electron potential can be expected in those lowdimensional materials where the width of the energy bands is smaller than ca. $1 \mathrm{eV}$. Suitable model systems falling into this category are lowdimensional organic and organometallic stacks, donor-acceptor complexes, biopolymers, solids with cluster substructures as well as the large class of Mott-insulators (transition metal oxides).

Some representative band structure data of the porphyrinato nickel(II) systems $\mathbf{2}$ and $\mathbf{3}$ have been analyzed as a function of the lattice sum dimension $j$ in order to demonstrate exemplarily the interrelation between geometrical variables (here: lattice spacing $c$ ) and the relative importance of the $k$ dependent one- and two-electron elements of the mean-field Hamiltonian. The $0.31 \AA$ elongation from $\mathrm{Ni}$ (TMP) $\mathbf{2}$ to $\mathrm{Ni}$ (OMTBP) $\mathbf{3}$ is accompanied by a "transition" from the tight-binding regime $(\varepsilon(k)$ curves determined by the one-electron part, see (23) and (24)) to the exchange-controlled regime ((25) and (26)). The exchange contributions beyond the direct neighbors are more or less hidden in solids with broad dispersion curves determined by the hopping matrix elements to the direct neighbors (covalent solids).

The present analysis had been restricted to the exchange influence in SCF HF CO variants defined via LCAO-type wave functions. Computational solid-state methods on the basis of local exchange potentials, however, should be also susceptible to the aforementioned phenomena. But it is obvious that the onset of strange exchange-dependent $\varepsilon(k)$ modifications is reduced in theoretical procedures where the "exact" nonlocal HF exchange has been replaced by simpler "local" $[\varrho(r)]^{1 / 3}$ approximations. As a qualitative rule of thumb, it can be assumed that the local exchange is about one order of magnitude smaller than the nonlocal HF potential. A further diminution of the hopping integrals is thus necessary before one-electron properties as discussed in the Sects. 3 and 5 can be observed.

The analysis in Sect. 6 has shown that band structures of narrow-band materials are neither reproduced by uncorrected HF bands nor by one-electron models of the WH or ET type. The width of a dispersion curve is overrated by the former CO procedures but is remarkably underestimated by effective one-electron Hamiltonians. Quasi-particle interactions (long-range and short-range correlations and relaxations) compensate partially the exchange- 
induced broadening of the $\varepsilon(k)$ curves. The $\Delta \varepsilon^{\mathrm{QP}}$ elements exceed nevertheless the dispersions derived by $\mathrm{WH}$ or EH methods. The HF exchange and the many-body part act into opposite directions; the net variation is always determined by the Fermi-correlation. The second mechanism responsible for the reduction of the QP band width is the electron (optical) phonon coupling which must be traced back to localization times $\tau$ of the charge carriers that are comparable with the frequencies of intramolecular vibrations; intermolecular modes, on the other side, can be neglected in weakly coupled materials. This phononic narrowing mechanism of the electronic bands should be always taken into account in systems with small $\Delta \varepsilon$ figures, irrespective of any theoretical details of the electronic Hamiltonian. On the basis of the data derived for $\mathrm{Ni}$ (OMTBP) 3 it is clear that QP band widths represent a theoretically reliable scheme for phononic corrections while $\Delta \varepsilon$ elements from WH variants are too small to allow for physically meaningful vibrational corrections.

The estimation of the antiferromagnetic coupling constant $J$ of 3 showed that relations as (4) are a suitable scheme for a comparison between experimental and calculated $\Delta \varepsilon$ elements. It is however necessary to adopt screened $t$ or $\Delta \varepsilon$ values that are corrected for QP and phononic interactions. It had been mentioned that (4) requires no a priori assumption on the analytic structure of the dispersion susceptible to the antiferromagnetic coupling. This degree of freedom is not part of those extrapolation procedures where experimental quantities of measurement (e.g., susceptibilities $\chi^{\mathrm{P}}$, plasma frequencies $\omega_{\mathrm{P}}$, thermoelectric power $\mathrm{S}$ ) are connected to

[1] J. Ladik and S. Suhai, in: Molecular Interactions, W. J. Orville-Thomas and H. Ratajack (ed.), Wiley Interscience, New York 1980; J.-M. André and J. Ladik, in: Recent Advances in the Quantum Theory of Polymers, Lecture Notes in Physics, Vol. 113, J.-M. André, J.-L. Brédas, J. Delhalle, J. Ladik, G. Leroy, and C. Moser (ed.), Springer Verlag, Berlin 1980.

[2] M. Kertész, Adv. Quantum Chem. 15, 161 (1982).

[3] M. Kertész, J. Koller, and A. Azman, in: Recent Advances in the Quantum Theory of Polymers, Lecture Notes in Physics, Vol. 113, J.-M. André, J.-L. Brédas, J. Delhalle, J. Ladik, G. Leroy, and C. Moser (ed.), Springer-Verlag, Berlin 1980.

[4] A. Karpfen, Int. J. Quantum Chem. 19, 1207 (1981).

[5] L. Piela, J.-M. André, J. G. Fripiat, and J. Delhalle, Chem. Phys. Letters 77, 143 (1981).

[6] S. Suhai, J. Chem. Phys. 73, 3843 (1980). electronic structure properties (e.g. $\Delta \varepsilon=4 t$ ) via idealized tight-binding relations based on the nearest neighbor's (hopping) interaction. The band structures presented in the sections 3 and 5 have shown that this simple "closed" expression is nonvalid in those solids where the width of a dispersion curve is prevailingly determined by the electronic exchange. A further consequence of this dominance is the breakdown of simple effective mass relations based on averaged parabolic wave vector connections:

$$
\begin{aligned}
& m=\hbar^{2} \pi^{2} /\left(2 c^{2} \Delta \varepsilon\right), \\
& c=\text { lattice spacing } .
\end{aligned}
$$

The symmetry properties in $k$-space supposed by (72) are not fulfilled in the discussed examples. The same problems are encountered if simplified formulas are employed to calculate group velocities.

To summarize: The present investigation has shown that the band structure properties of solids with narrow dispersions are largely influenced by the nonlocal HF exchange. The divergent decay properties of the kinetic hopping matrix elements and the electronic exchange lead to one-electron properties that differ substantially from the band structures of highly covalent solids which are very well described by classical tight-binding interactions.

\section{Acknowledgements}

This work has been supported by the Stiftung Volkswagenwerk and by the Max-Planck-Gesellschaft. The author wants to express his thanks to Prof. P. Fulde and Dipl.-Phys. F. Pfirsch for various useful and stimulating discussions. The assistance of Mrs. I. Grimmer in the preparation of the manuscript is gratefully acknowledged.

[7] P. R. Surján, M. Kertész, A. Karpfen, and J. Koller, Phys. Rev. B 27, 7583 (1983).

[8] R. Dovesi, Int. J. Quantum Chem. 26, 197 (1984).

[9] F. E. Harris, in: Theoretical Chemistry, Vol. 1, H. Eyring and D. Hendersen (ed.), Academic Press, New York 1975 .

[10] J. Cižek, G. Biczó, and J. Ladik, Theor. Chim. Acta 8, 175 (1967).

[11] I. I. Ukrainski, Theor. Chim. Acta 38, 139 (1975).

[12] F. Seitz, Modern Theory of Solids, McGraw-Hill, New York 1940; J. C. Slater, Insulators, Semiconductors and Metals, McGraw-Hill, New York 1967.

[13] H. J. Monkhorst, Phys. Rev. B 20, 1504 (1979).

[14] H. J. Monkhorst and M. Kertész, Phys. Rev. B 24, 3015 (1981).

[15] H. Fujita and A. Imamura, J. Chem. Phys. 53, 4555 (1970). 
[16] M. Kertész, Acta Phys. Acad. Sci. Hung. 41, 107 (1976).

[17] J.-L. Brédas, in: Recent Advances in the Quantum Theory of Polymers, Lecture Notes in Physics, Vol. 113, J.-M. André, J.-L. Brédas, J. Delhalle, J. Ladik, G. Leroy, and C. Moser (ed.), Springer-Verlag, Berlin 1980.

[18] L. Piela and J. Delhalle, Int. J. Quantum Chem. 13, 605 (1978); J. Delhalle, L. Piela, J.-L. Brédas, and J.-M. André, Phys. Rev. B 22, 6254 (1980).

[19] A. Karpfen and J. Petkov, Theor. Chim. Acta 53, 65 (1979); A. Karpfen and R. Höller, Sol. State Commun. 37, 179 (1981).

[20] J. S. Miller (ed.), Extended Linear Chain Compounds, Plenum Press, New York 1982.

[21] J. A. Ibers, L. J. Pace, J. Martinsen, and B. M. Hoffman, Struct. Bonding 50, 1 (1982); B. M. Hoffman and J. A. Ibers, Acc. Chem. Res. 16, 15 (1983).

[22] M. C. Böhm, Theor. Chim. Acta 62, 373 (1983).

[23] M. C. Böhm, J. Phys. C: Sol. State Phys. 16, 1631 (1983).

[24] M. C. Böhm, J. Phys. C: Sol. State Phys. 17, 2091 (1984).

[25] M. C. Böhm, Phys. Rev. B 28, 6914 (1983).

[26] M. C. Böhm, Int. J. Quantum Chem. 25, 817 (1984).

[27] M. C. Böhm, J. Chem. Phys. 81, 855 (1984).

[28] M. C. Böhm, Physica 124 B, 203 (1984).

[29] M. C. Böhm, Physica 125 B, 1 (1984).

[30] M. C. Böhm, Theor. Chim. Acta 62, 351 (1983).

[31] M. C. Böhm and R. Gleiter, Theor. Chim. Acta 59, 127,153 (1981).

[32] S. Alvarez and E. Canadell, Sol. State Commun. 50, 141 (1984).

[33] E. Canadell and S. Alvarez, Inorg. Chem. 23, 573 (1984).

[34] J. Martinsen, L. J. Pace, T. E. Phillips, B. M. Hoffman, and J. A. Ibers, J. Amer. Chem. Soc. 104, 83 (1982).

[35] L. J. Pace, J. Martinsen, A. Ulman, B. M. Hoffman, and J. A. Ibers, J. Amer. Chem. Soc. 105, 2612 (1983).

[36] T. E. Phillips, R. P. Scaringe, B. M. Hoffman, and J. A. Ibers, J. Amer. Chem. Soc. 102, 3435 (1980).

[37] B. M. Hoffman, T. E. Phillips, and Z. G. Soos, Sol. State Commun. 33, 51 (1980).

[38] J. Hubbard, Proc. Roy. Soc. London Ser. A 276, 238 (1963); 277, 237 (1963); 281, 401 (1964).

[39] Z. G. Soos, Ann. Rev. Phys. Chem. 25, 121 (1974); Z. G. Soos and D. J. Klein, in: Molecular Association, R. Foster (ed.), Academic Press, New York 1975.

[40] J. B. Torrance, Acc. Chem. Res. 12, 79 (1979).

[41] G. Beni, T. Holstein, and P. Pincus, Phys. Rev. B 8, 312 (1973); D. J. Klein, Phys. Rev. B 8, 3452 (1973).

[42] H. Shiba, Phys. Rev. B6, 930 (1972); J. B. Torrance, Y. Tomkiewicz, and B. D. Silverman, Phys. Rev. B 15, 4738 (1977).

[43] L. C. Isett, Phys. Rev. B 18, 439 (1978).

[44] R. J. Elliot and A. F. Gibson, An Introduction to Solid State Physics and its Applications, Macmillan Press, London 1974.

[45] B. N. Diel, T. Inabe, J. W. Lyding, K. F. Schoch, Jr., C. R. Kannewurf, and T. J. Marks, J. Amer. Chem. Soc. 105, 1551 (1983).

[46] P. M. Chaikin, R. L. Greene, S. Etemad, and E. Engler, Phys. Rev. B 13, 1627 (1976).

[47] P. M. Chaikin, G. Grüner, T. F. Shegolev, and E. B. Yagubskii, Solid State Commun. 32, 1211 (1979).

[48] M. C. Böhm, Phys. Letters 102 A, 121 (1984).

[49] M. C. Böhm, Chem. Phys. Letters 107, 322 (1984).

[50] M. C. Böhm, manuscript in preparation.
[51] T. E. Peacock and R. McWeeny, Proc. Phys. Soc. London 74, 385 (1959).

[52] G. del Re, J. Ladik, and G. Biczó, Phys. Rev. 155 , 997 (1967); J.-M. André, L. Gouverneur, and G. Leroy, Int. J. Quantum Chem. 1, 997 (1967).

[53] J. Delhalle, Int. J. Quantum Chem. 8, 201 (1974).

[54] J. Delhalle, in: Electronic Structure of Polymers and Molecular Crystals, J.-M. André, and J. Ladik (ed.), Plenum Press, New York 1975.

[55] W. Gautschi, Num. Math. 18, 373 (1972).

[56] F. Pfirsch and M. C. Böhm, Chem. Phys. Letters, in press.

[57] R. Ramirez, F. Pfirsch, and M.C. Böhm, unpublished results.

[58] L. Salem, Proc. Cambridge Philos. Soc. 57, 393 (1961).

[59] O. K. Andersen, Phys. Rev. B 12, 3060 (1975); R. O. Jones, Phys. Bl. 40, 149 (1984).

[60] M. C. Böhm, Z. Phys. B: Condensed Matter 56, 99 (1984).

[61] M. C. Böhm, Z. Naturforsch. 39 a, 807 (1984).

[62] D. R. Hartree, The Calculation of Atomic Structure, Wiley Interscience, New York 1957.

[63] L. E. Sutton (ed.), Tables of Interatomic Distances and Configuration in Molecules and Ions, Spec. Publ. 18, The Chemical Society, London 1965.

[64] R. S. Mulliken, J. Chem. Phys. 23, 1833 (1955).

[65] Y. Toyozawa, Prog. Theor. Phys. (Tokyo) 12, 421 (1954).

[66] J. Hermanson, Phys. Rev. B6, 2427 (1972); A. W. Overhauser, Phys. Rev. B3, 1888 (1971).

[67] A. B. Kunz, Phys. Rev. B6, 606 (1972); D. J. Mickish, A. B. Kunz, and T. C. Collins, Phys. Rev. B 9, 4461 (1974); A. B. Kunz, Phys. Rev. B 12, 5890 (1975).

[68] S. T. Pantelides, D. J. Mickish, and A. B. Kunz, Phys. Rev. B 10, 2602 (1974).

[69] S. Horsch, P. Horsch, and P. Fulde, Phys. Rev. B 28 , 5977 (1983); S. Horsch, P. Horsch, and P. Fulde, Phys. Rev. B 29, 1870 (1984).

[70] W. Hanke, H. J. Mattausch, and G. Strinati, in: Electron Correlations in Solids, Molecules and Atoms, J. T. Devreese and F. Brosens (ed.), Plenum Press, New York 1983.

[71] S. Suhai, Phys. Rev. B 27, 3506 (1983); S. Suhai, Int. J. Quantum Chem. 23, 1239 (1983).

[72] M. C. Böhm, Int. J. Quantum Chem., in press.

[73] M. C. Böhm, Phys. stat. Sol. (b), 127, 209 (1985).

[74] B. T. Pickup and O. Goscinski, Mol. Phys. 26, 1013 (1973); G. Born, H. A. Kurtz, and Y. Öhrn, J. Chem. Phys. 68, 74 (1978).

[75] M. C. Böhm, J. Chem. Phys. 78, 7044 (1983); M. C. Böhm, J. Phys. B: At. Mol. Phys. 17, 3103 (1984).

[76] F. J. Dyson, Phys. Rev. 75, 486 (1949)

[77] C. J. Schramm, R. P. Scaringe, D. R. Stojakovic, B. M. Hoffman, J. A. Ibers, and T. J. Marks, J. Amer. Chem. Soc. 102, 6702 (1980).

[78] M. Gouterman, G. H. Wagnière, and L. C. Snyder, J. Mol. Spectrosc. 11, 108 (1963); L. Edwards and M. Gouterman, J. Mol. Spectrosc. 33, 292 (1970).

[79] S. Suhai, Phys. Letters 62 A, 185 (1977); S. Suhai, in: Quantum Theory of Polymers, J.-M. André, J. Delhalle, and J. Ladik (ed.), D. Reidel Publ. Co., Dordrecht-Boston 1978.

[80] N. O. Lipari, C. B. Duke, and L. Pietronero, J. Chem. Phys. 65, 1165 (1976); N. O. Lipari, C. B. Duke, R. Bozio, A. Girlando, C. Pencile, and A. Padva, Chem. Phys. Letters 44, 236 (1976). 